Review paper

\title{
Dental cone beam CT: An updated review
}

\author{
Touko Kaasalainen $^{\text {a, *, Marja Ekholm }}{ }^{\text {b, c }}$, Teemu Siiskonen ${ }^{\text {d, }}$, Mika Kortesniemi ${ }^{\text {a }}$ \\ ${ }^{a}$ HUS Diagnostic Center, Radiology, Helsinki University and Helsinki University Hospital, P.O. Box 340, Haartmaninkatu 4, o0290 Helsinki, Finland \\ ${ }^{\mathrm{b}}$ Institute of Dentistry, University of Turku, Lemminkäisenkatu 2, 20520 Turku, Finland \\ ${ }^{\mathrm{c}}$ South West Finland Imaging Center, Turku University Hospital, Lemminkäisenkatu 2, 20520 Turku, Finland \\ ${ }^{\mathrm{d}}$ Radiation Practices Regulation, Radiation and Nuclear Safety Authority - STUK, P.O. Box 14, FI-00881 Helsinki, Finland
}

\section{A R T I C L E I N F O}

\section{Keywords:}

Dental

CBCT

Characteristics

Dosimetry

Image quality

Guidelines

Development

Cone beam CT

Imaging

\begin{abstract}
A B S T R A C T
Cone beam computed tomography (СBCT) is a diverse 3D x-ray imaging technique that has gained significant popularity in dental radiology in the last two decades. CBCT overcomes the limitations of traditional twodimensional dental imaging and enables accurate depiction of multiplanar details of maxillofacial bony structures and surrounding soft tissues. In this review article, we provide an updated status on dental CBCT imaging and summarise the technical features of currently used CBCT scanner models, extending to recent developments in scanner technology, clinical aspects, and regulatory perspectives on dose optimisation, dosimetry, and diagnostic reference levels. We also consider the outlook of potential techniques along with issues that should be resolved in providing clinically more effective CBCT examinations that are optimised for the benefit of the patient.
\end{abstract}

\section{Introduction}

Digitalisation of medical imaging has brought major transformations in dental radiology with restructuring of the imaging process (image acquisition, post-processing, and image review methods), which enables more efficient image archiving and communication (PACS systems) [1]. Dental x-ray imaging forms a variable combination of radiological imaging techniques. The methods include 2D imaging (intraoral, cephalometric radiography, panoramic x-ray) and 3D imaging (multislice computed tomography [CT] and cone beam CT [CBCT]) techniques [2-6].

Traditional dental x-ray imaging has for decades applied 2D image acquisition and panoramic mode. However, superposition of true volumetric anatomy in these overlaid 2D images conceals potentially important clinical findings that would require multiplanar visualisation to reveal local and typically focal findings in any possible orientation $[3,5,7,8]$. More specifically, due to human anatomy, projection radiography and panoramic imaging of the dental area are mainly performed from the mediolateral direction (from outside of the jaw towards inside of the oral cavity). As such, the labiopalatal (through lips to tongue) and buccolingual (through cheek to tongue) structures are overlaid and their dimensional nature can therefore only be subjectively estimated. Many clinically relevant structures, such as dental roots related to surrounding soft tissue and alveolar bony anatomy, are not adequately represented in traditional images and may lead to missed clinical findings, e.g. periapical lesions located on the tip of the root canal [9]. There are also additional complications in implementing imaging settings of the dental area. Parallel projections require a well-defined and maintained directional setting of the radiography equipment to provide accurate geometric representation of the dental structures. Due to these limitations and acknowledging the complicated 3D anatomy of the dental area, 3D imaging has increased in popularity to provide an accurate depiction of the multiplanar details of maxillofacial bone structures and surrounding soft tissues [5,10].

Rapid progress in medical imaging physics and technology, involving several hardware and computational methods, has overcome many challenges to achieve 3D dental imaging (known as CBCT) and to offer tools to improve 3D image quality in terms of spatial resolution, contrast, and anatomical coverage [3,5]. CBCT is also known as digital volume tomography, which refers to representation of image data in digital forms, and most essentially, depiction of anatomy in three dimensions. The development of CBCT began in 1995 when Italian developers Attilio Tacconi and Piero Mozzo introduced the first dental CBCT model, the NewTom DVT 9000 [11]. The commercial launch of the device came a few years later, along with similar equipment developed by Arai et al. in 1999 [12].

\footnotetext{
* Corresponding author at: HUS Medical Imaging Center, Helsinki University Hospital, P.O. Box 340 (Haartmaninkatu 4), 00290 Helsinki, Finland.

E-mail address: touko.kaasalainen@hus.fi (T. Kaasalainen).
} 
The principle of CBCT adapts the basic CT technique where the $\mathrm{x}$-ray tube exposes the patient from one side and an imaging detector measures the attenuated $x$-rays on the other side of the patient, while both the $x$-ray tube and detector rotate around the patient [13] (Fig. 1). The exposure during the rotational scan can utilise either continuous exposure or pulsed exposure; the latter is increasingly applied in dental CBCT [14]. Pulsed exposure reduces the effective exposure time and reduces the dose to the patient [5]. The attenuation of the $\mathrm{x}$-rays follows the basic physical interactions between radiation and atomic composition of the patient anatomy in the exposed field of view (FOV). These physical interactions include mainly photoelectric effect and Compton scattering, which set the basic balance for the contrast representation of the images. The contrast information in the image signal is mostly contributed by the photoelectric effect, whose contribution diminishes steeply as the x-ray photon energy increases. Therefore, the image contrast decreases when higher tube voltages are applied. At the same time, the contribution from scattering increases along with the spectral shift towards higher quantum energy $\mathrm{x}$-rays [15].

3D image reconstruction is the core part of CT imaging in the computational aspect. Image reconstruction attempts to solve the mathematical inverse problem, namely how can the patient image (which is the 3D map of material-specific attenuation values in the patient) be deduced from the x-ray shadow signal acquired during the exposure? Traditional analytical reconstruction algorithms have been used in typical mainstream CBCT scanners. These algorithms are typically based on the approximate Radon inverse transformation introduced by Feldkamp et al. in 1984 [16]. In recent years, iterative reconstruction algorithms have been increasingly applied due to the potential for image quality improvements and artefact reduction $[17,18]$. The iterative image reconstruction approach may also mitigate one of the most pertinent challenges in CBCT, which is sensitivity to motion artefacts due to the relatively long scan time [19], which is inevitable with typical CBCT C-arm type gantry design. Recently, artificial intelligence (AI) based deep-learning (DL) methods have been developed as the latest advancement in image reconstruction [20,21]. DL image reconstruction methods utilise large amounts of typical clinical or technical phantom image data (or both) to solve the reconstruction problem and to provide improved image quality. The challenge in these new AI-based reconstruction algorithms is that the learning data should be sufficiently representative to cover highly variable patient anatomy and contrast cases. Thus, the reconstruction method should be generalisable to all imaging cases and provide reliable and accurate image quality for all patients [21].

The distinctive exposure geometry utilised in CBCT scanners is characterised by the cone- or pyramid-shaped x-ray beam, which is directed on the selected dental part contained in the scan FOV. In this instance, the cone refers to a beam that is around the same dimensional size in the vertical $(\mathrm{z})$ direction as compared to axial $(\mathrm{x}, \mathrm{y})$ direction (Fig. 1). Modern CBCT scanners use flat-panel detectors (FPD) structured as a pixel matrix of amorphous silicon thin-film transistors (TFT) or complementary metal-oxide semiconductors (CMOS) to capture the image signal. Both TFT and CMOS detector techniques are based on the indirect conversion principle, where the absorbed $\mathrm{x}$-rays at the detector scintillation layer are first converted into light photons that are detected by photodiodes and finally read from the whole photodiode pixel matrix. By this process, one raw-data projection view from a single angular direction is acquired to be compiled with other projections in the CBCT raw data. The scintillation material that is used to absorb the x-rays and convert their energy into light photons consists of thallium-doped caesium iodide (CsI:Tl) or terbium-activated gadolinium oxysulfide $\left(\mathrm{Gd}_{2} \mathrm{O}_{2} \mathrm{~S}: \mathrm{Tb}\right)$. FPD technology provides benefits for imaging, including high spatial resolution, large dynamic range of signal levels, slim structure, and more streamlined imaging chain when compared with traditional image intensifier (II) or charge-coupled device (CCD) based image detectors, which have become obsolete in CBCT equipment $[3,22,23]$. CMOS technology provides even higher resolution, faster image readout, and lower electronic noise in comparison to current amorphous silicon detector models, which have potential for more optimised scans and improved clinical image quality [24].

Acquired raw data in the form of projection x-ray images go through various pre-processing steps before they can be used for image reconstruction. This pre-processing considers detector features and exposure factors, which corrects for different limitations and inherent variabilities of the detector. These include dark current adjustments of the detector in addition to gain and pixel defects by performing gain and offset correction. A series of raw data projections are acquired at a rapid rate during the rotational CBCT scan. Such a high frame rate may also leave partial signal from the previous projection image in the subsequent projection image readout. This possible latent image signal must be erased from the projection image data by applying afterglow correction. Exposure factors involve x-ray beam properties, which include x-ray spectrum (affected by tube voltage and beam pre-filtration), scatter distribution at the detector surface (depending on spectrum, scan, and patient geometry), focal spot size, and focus-to-detector distance (affecting image sharpness and tube output). Exposure factors also extend to detector response (based on the specific detector and readout electronics design), among other physical features of the scan $[13,25]$.

One of the primary economic benefits of CBCT equipment when

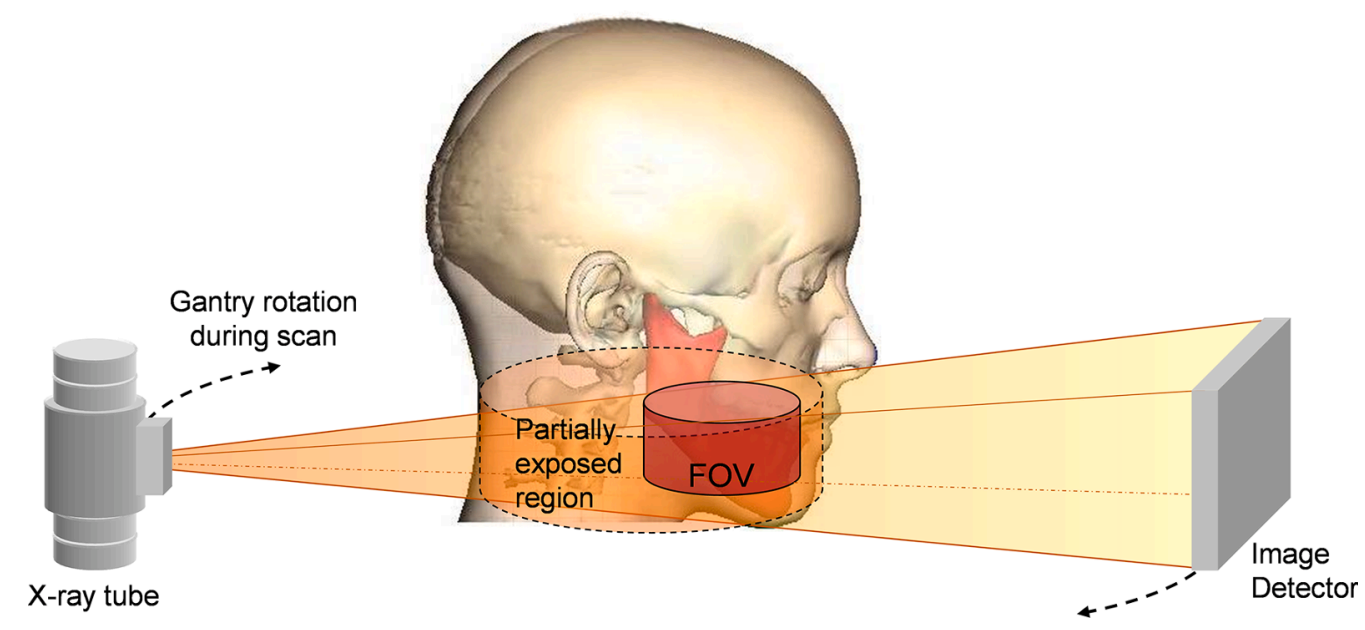

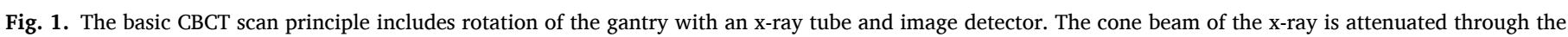

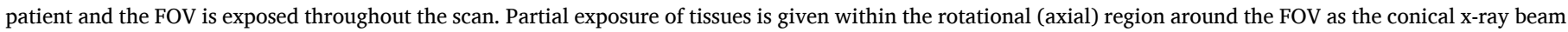

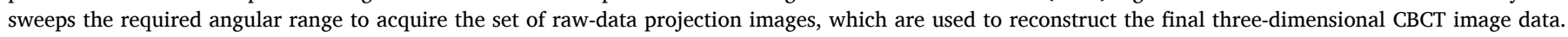


compared with multi-slice CT scanners is related to the significantly lower requirement of $\mathrm{x}$-ray output in CBCT scanners, which places it typically at the same level of $x$-ray tube current and spectral output as dental panoramic x-ray scanners [10]. The footprint for CBCT installation (with or without cephalostat) requires only a few square meters of room space (similar to panoramic equipment) and therefore the transition from panoramic imaging to CBCT does not require major alterations in dental facilities. Most CBCT gantry designs involve patient positioning in a standing or sitting position (in contrast to supine lying position), which also helps reduce equipment footprint $[5,14]$. Indeed, the gantry of vertical CBCT follows the structure of the panoramic device and may combine both functionalities (and also cephalometric imaging) in addition to providing an advanced digital detector, image reconstruction and processing workstation, and related software package. The electrical supply and HPAC (heating, plumbing, air-conditioning) factors required by the CBCT scanner are similar to panoramic x-ray equipment, and therefore does not require further renovations.

Medical imaging examinations using $\mathrm{x}$-rays must always conform to the main radiation protection principles, where the minimisation of the exposed anatomical region is a pivotal part of the optimisation of the medical exposure. Furthermore, minimisation of the exposed volume also improves image quality, as scattered radiation, which reduces image contrast, is mitigated with a smaller exposed FOV [13]. In dental CBCT imaging, varying FOV selection is an essential characteristic in all modern scanners, which enables indication-specific optimisation of image FOV and also affects radiation exposure to the patient. User adjustments in FOV size and image quality settings also determine the voxel size used in the image acquisition. Acquired voxel size is directly linked to observed spatial resolution according to the sampling principles. As such, small details in dental structures are represented more accurately with smaller voxel sizes. However, smaller voxel sizes require higher radiation doses to achieve the same signal-to-noise ratio (SNR) when compared with larger voxel size. According to Poisson statistics, the relative dose increase to maintain SNR is inversely proportional to the square root of the relative voxel volume decrease. For example, a four times larger voxel volume enables halving the dose with the same image noise $[5,15]$.

One of the main strengths in CBCT imaging is the possibility to create various $3 \mathrm{D}$ visualisations and multi-planar reformats of the volumetric image data. This possibility is accentuated by production of isotropic or nearly isotropic image data, e.g. creating voxels where the voxel dimensional length is approximately the same in all three directions ( $\mathrm{x}$, $\mathrm{y}$, and $\mathrm{z}$ ) [5]. Isotropic image data enable similar image quality regardless of the projected direction of the reformatted slices, which significantly aids interpretation and review of the complex 3D anatomy of the jaw region. There is also a wide range of post-processing options to utilise 3D image data beyond cross-sectional views. These options cover traditional dental, virtual panoramic (curved multiplanar reformat) or cephalometric views, or bilateral multiplanar projections of the tempomandibular joint [10]. All CT imaging techniques, including dental CBCT, are inherently accurate in geometry. This means that the reconstructed images represent 3D x-ray attenuation distribution of the object and do not encompass diverging projection geometry with varying magnifying ratio of superimposed structures across the central beam axis, as in traditional dental imaging or in the acquired CBCT raw-data projections (before reconstruction). Therefore, the linear measurements can be performed accurately and reproducibly from multiplanar CBCT image data [26].

Although dental CBCT can produce superior spatial resolution with the smallest voxel sizes often $<100 \mu \mathrm{m}$, the low-contrast resolution to describe soft tissue structures is limited due to various physical and technical factors [10]. These factors include inevitable scattered radiation that reduces the contrast in the projection raw data and consequently also the final reconstructed image data. Scatter also causes streak artefacts emerging from metal surfaces. Another factor is beam hardening, which is caused by the higher attenuation of softer (lower quantum energy) $\mathrm{x}$-rays in the polychromatic $\mathrm{x}$-ray beam due to patient structures. Beam hardening increases the mean energy of the transmitted x-ray spectrum [15]. Thus, the resulting image data corresponding to the beam-hardened projections represent lower attenuation than the real attenuation in the patient. Softer x-rays may be completely depleted in this process (called photon starvation), and this is also related to artefact appearance. Thus, the beam-hardening effect is accentuated in the presence of extensive metallic restorations, dental implants, and orthopedic hardware [27]. Additional artefacts are produced by undersampling, which are related to large density differences between metals and the surrounding lower attenuating structures, causing thin white streaks originating from the metal target. Thus, streak artefacts are caused by multiple sources, including scatter, beam hardening, and sampling issues [28]. Low-contrast resolution of CBCT is also decreased by truncation effects, which are caused by smaller FOVs than the whole surrounding anatomy [29,30] (Fig. 1). In CBCT scans, the rotating $\mathrm{x}$-ray beam sweeps through all attenuating structures of the patient regardless of if they are inside or outside the FOV. Thus, extra FOV structures also cause decreases in signal in the acquired raw-data projection images, which appear as contrast deviation in the reconstructed images. Truncation artefacts appear as a band of lighter than expected gray scale voxels on the edge of the FOV if not corrected [29]. Another cause of the lowered soft-tissue contrast accuracy in dental CBCT is due to the lower number (typically hundreds) of raw-data projections when compared with multi-slice CT acquisitions (typically thousands of projections). Nevertheless, a sharp 3D representation of bony tissues is the key strength of dental CBCT imaging, and thus the limited soft-tissue contrast remains a tolerable limitation that is mitigated through development of new scanner hardware and image reconstruction methods [5].

The required spatial resolution is driven by the smallest clinical details that must be distinguished to make reliable clinical decisions. For example, a periodontal ligament gap in the range of $100 \mu \mathrm{m}(0.1 \mathrm{~mm})$ must be seen in periodontal CBCT applications for reliable diagnosis $[31,32]$. Spatial resolution is not only linked to the voxel size. Focal spot size, contrast resolution and range, detector motion unsharpness, detector fill factor, number of raw-data projections per rotation, noise performance, and reconstruction technique also clearly impact spatial resolution at the technical level. However, patient movement poses a significant challenge to spatial resolution [5]. Therefore, the observed spatial resolution, achieved in technical phantom scans in QA testing, is not reached in clinical scans with patients. Even if the patient would be perfectly fixated to the imaging position at the CBCT scan isocentre, hemodynamic pulsation produces periodical motion, which also extends to maxillofacial anatomy and scanned dental structures. This cardiac pulsation alone can create spatial unsharpness of 80 to $90 \mu \mathrm{m}$. When all relevant factors in clinical CBCT scans are considered, the motion blur is at the order of $500 \mu \mathrm{m}$ (corresponding to 1 line pair per $\mathrm{mm}$ ), thereby resulting in notably larger spatial uncertainty than the small voxel size $[32,33]$. In practice, involuntary patient motion can add even more unsharpness to the final image if not corrected. Therefore, different motion correction algorithms have been incorporated to several recent CBCT scanner models to overcome this image quality challenge and consequently offer sharper clinical images [34].

The main exposure parameters used in CBCT scans present the same general rules on image quality and radiation exposure of the patient as in other medical imaging modalities that use $\mathrm{x}$-rays. Tube voltage and prefiltration adjust the spectrum of $x$-rays, which in turn affects the image contrast (through balance between photoelectric effect and Compton scatter) and radiation dose. Tube current and exposure time are directly proportional to the tube output and the radiation dose, which affect image noise level according to Poisson statistics (noise level is inversely proportional to the square root of the dose). Dose is affected by tube voltage roughly as proportional to the voltage value squared [15].

In this review, we provide an updated summary of the current status of dental CBCT imaging, availability of different scanner models, recent 
developments in scanner technology, and clinical aspects. We also address the regulatory perspective regarding dose optimisation, dosimetry, and diagnostic reference levels. This review can be regarded as an update to our previous review article published in 2015 [5].

\section{Technical characteristics of current dental CBCT scanners}

The last 5 to 10 years have seen a steady development of incremental technical CBCT features; current technology seems to be relatively static (see our previous CBCT review article [5]). Even so, new CBCT equipment models are continuously being developed and released. The systems available on the market and used in clinical work vary remarkably in their imaging features and capabilities. The end users can select a proper CBCT device for their clinical needs from a wide range of different CBCT systems. The technical properties of the currently available scanners are summarised in Table 1 and are discussed in the text. To gather the data, an email inquiry was sent to each CBCT manufacturer with a request for brochures and technical data sheets of various $\mathrm{CBCT}$ devices. As some of the manufacturers did not respond to the inquiry or were not willing to share technical information for this review article, the data were completed as comprehensively as possible by searching for technical specifications from the manufacturers' brochures and manuals and from other information available on the internet (mostly from manufacturers' webpages). Recent scientific literature was also searched with the keywords "CBCT technology" and "Dental CBCT" to obtain updates on the essential technical and review articles published since our previous review. A recent review article from Gaêta-Araujo et al. [14] comprehensively summarised features of currently and formerly available CBCT equipment from 1996 to 2019. Their study included altogether 279 different CBCT models from 47 manufacturers, of which 203 systems were reported to be still available in 2020 .

\subsection{General features}

CBCT systems are typically divided into three different categories according to FOV size: (1) large FOV ( $>15 \mathrm{~cm}$ maximum scan volume height), (2) medium FOV (from 10 to $15 \mathrm{~cm}$ field height), and (3) small FOV ( $\leq 10 \mathrm{~cm}$ field height) [35]. Most of the devices also allow panoramic imaging, and with an additional arm, cephalometric imaging with the same unit. A few CBCT systems also have 3D photography capability for assisting pre-operative treatment planning and follow up. Moreover, some of the dental CBCT systems currently available may also be used for orthopaedic CBCT imaging. All currently available systems have a dose display (as required by regulations) that are mostly based on computational estimations. A major portion of the CBCT systems present dose after each exposure using a dose quantity of dose-area product (DAP) or kerma-air product (KAP or $\mathrm{P}_{\mathrm{KA}}$, similar to DAP in diagnostic radiology), although a few systems use computed tomography dose index (CTDI) for this purpose. Some systems report both dose quantities.

\subsection{Gantry size and type}

The size and geometry of CBCT systems vary mostly on the technique used for patient positioning. The weight of the systems ranges from 67 $\mathrm{kg}$ up to $1050 \mathrm{~kg}$. Most devices allow scanning both in standing and sitting positions, while a few systems utilise scanning only in a supine position. Gaêta-Araujo et al. [14] reported that $80 \%$ of the CBCT devices were either wall- or floor-mounted standing position systems (mostly with wheelchair access), while supine lying position was used only in $3 \%$ of the devices. While the standing position is the most used for dental CBCT scanning, it is vulnerable to patient movements especially when sufficient head fixation tools are not used. To minimise patient motionrelated artefacts, standing devices can typically also be used in a sitting position due to motorized columns of the systems, allowing vertical movements of x-ray tube and detector head. This also allows access to wheelchair patients. Some of the vendors also provide motioncorrection algorithms and other solutions to improve image quality regardless of patient movement. For example, 3Shape X1 Scancomfort device (3Shape A/S, Copenhagen, Denmark) uses head-tracking technology to measure patient movement during the scanning, and reconstruction software then readjusts data for any motion to deliver sharp images. Although standing-position systems are frequently used, seatedand supine-position systems may allow a more comfortable imaging experience to a patient and thereby reduce motion-related artefacts. Some of the seated systems also have a vertical column, which provides access to wheelchair patients. Supine lying position equipment require significantly larger room dimensions than the standing and seated systems and may also have other essential requirements for the space, possibly due to the greater weight of such systems.

\subsection{Field of view (FOV)}

The FOVs available in dental and maxillofacial CBCT systems vary from FOVs suitable for a single jaw or a few teeth (D $\times$ H usually $4 \times 4$ $\mathrm{cm}$ or $5 \times 5 \mathrm{~cm}$, or from $2 \times 2 \mathrm{~cm}$ in freely adjustable FOV systems) to full craniofacial imaging (from approximately $15 \times 15 \mathrm{~cm}$ up to $26 \times 30$ $\mathrm{cm}$ or $17 \times 32 \mathrm{~cm}$ ). The FOV dimensions depend on the detector size and shape, beam projection geometry, and the ability to collimate the beam [22]. While most of the devices utilise either one or a few prefixed FOVs (up to 23 different FOVs) planned for different indications, a user can freely adjust the FOV in the certain limits in both the vertical and the horizontal cross-sectional volumes in some equipment. Additionally, some CBCT devices allow stitching of the adjacent 3D volumes either in the horizontal or vertical direction, or both, resulting in larger FOVs. We want to emphasise that the FOV along with the voxel size is one of the most important parameters that affects patient radiation dose and image quality and should be selected according to the clinical task.

\subsection{Data collection during the scan}

For CBCT data reconstruction, a scan arc of at least $180^{\circ}$ should be used to acquire the projection images [22]. Most currently available dental CBCT systems use a rotation angle of $360^{\circ}$ for data acquisition; the range varies between $180^{\circ}$ and $540^{\circ}$ depending on the system. In a few devices, it is possible to select two or three different rotation angles for scanning, which allows reduced radiation exposure to the patient. While the lower angular range typically helps reduce patient dose [36], this may also reduce image quality. Moreover, some CBCT units utilise offset of the central ray of the beam from the rotational centre of the device to expand the width of the FOV, effectively imaging half of the field through the first $180^{\circ}$ of rotation and the adjacent half through the second $180^{\circ}$ of rotation. This strategy allows reduction in the size and expense of the flat panel detector. The number of projection images per rotation varies remarkably between devices. This may also affect image quality to some extent as the increased number of samples theoretically provides higher image quality. The grayscale depth of the image data varies between 12 and 16 bits, and almost every new CBCT model utilises either 14 bits or 16 bits.

\subsection{Voxel and focal spot size}

Most modern dental CBCT systems allow selection of the voxel size used according to clinical requirements. Since our previous dental CBCT review article in 2015 [5], the voxel sizes available in the devices have tended to be even smaller, possibly due to improved image reconstruction algorithms, image noise reduction, and patient movement correction methods. The smallest voxel size currently available is $50 \mu \mathrm{m}$ (Smart3D-X scanner, LargeV Instrument Corp Ltd., Beijing, China), while the largest possible voxel size is $600 \mu \mathrm{m}$ (Planmeca CBCT systems, Planmeca Oy, Helsinki, Finland). The voxel size is related to spatial resolution. Therefore, smaller voxel sizes enable higher spatial 
Table 1

Technical characteristics of the currently available dentomaxillofacial CBCT equipment.

\begin{tabular}{|c|c|c|c|c|c|c|c|c|c|c|c|c|c|c|c|c|c|c|c|}
\hline 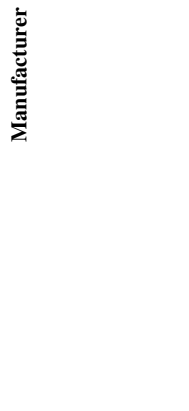 & 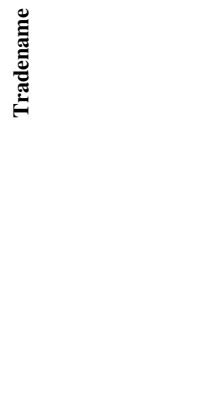 & 产 & 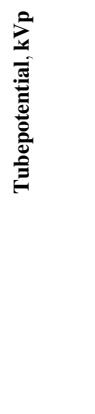 & 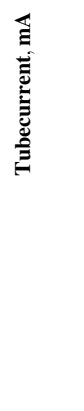 & 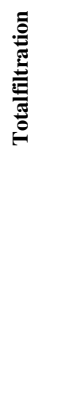 & 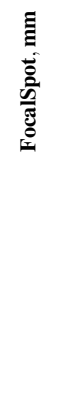 & 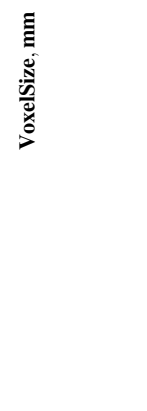 & 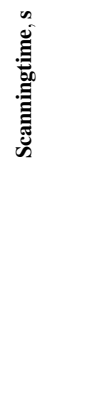 & 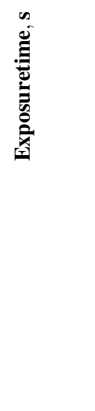 & 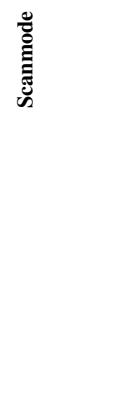 & 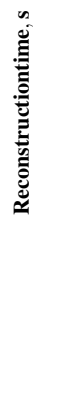 & 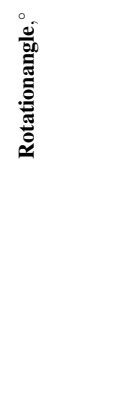 & 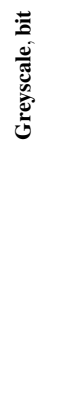 & 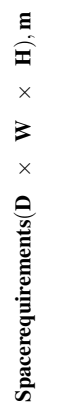 & 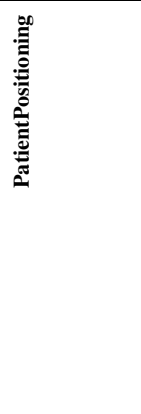 & $\begin{array}{l}5 \\
\bar{\Xi} \\
\times \\
0 \\
0\end{array}$ & 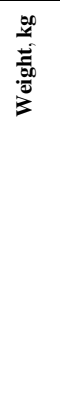 & 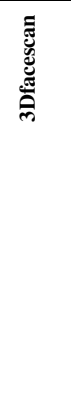 & 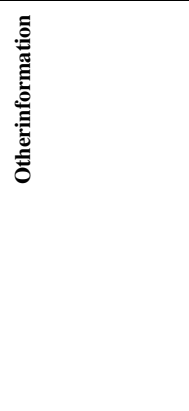 \\
\hline 3Shape & X1 Scancomfort & $\mathrm{N} / \mathrm{A}$ & $60-90$ & $4-12$ & $\mathrm{~N} / \mathrm{A}$ & $\begin{array}{l}\mathrm{N} / \\
\mathrm{A}\end{array}$ & $0.075-0.400$ & $\mathrm{~N} / \mathrm{A}$ & 5.8 & $\mathrm{~N} / \mathrm{A}$ & $<300$ & $\begin{array}{l}180 / \\
360\end{array}$ & N/A & $\begin{array}{l}1.60 \\
\times \\
1.30 \\
\times \\
2.30\end{array}$ & $\begin{array}{l}\text { Seated / } \\
\text { Standing / } \\
\text { Wheelchair }\end{array}$ & $\begin{array}{l}\text { freely } \\
\text { adjustable } \\
\text { FOV from } 2 \\
\times 2 \text { to } 15 \times \\
15 \text { (standard: } \\
2 \times 2-8 \times 8 \text {; } \\
\text { option: } 15 \times \\
8 ; 15 \times 15 \text { ) }\end{array}$ & 230 & Yes & $\begin{array}{l}\text { Iterative } \\
\text { reconstruction, } \\
\text { 3D head tracking } \\
\text { system, scout } \\
\text { image, free head } \\
\text { positioning }\end{array}$ \\
\hline Acteon Group & X-Mind Prime 3D & $\begin{array}{l}\text { CsI / } \\
\text { CMOS }\end{array}$ & $60-86$ & $2-12.5$ & $\begin{array}{l}2.5 \\
\text { mmAl }\end{array}$ & 0.5 & $0.088-$ & N/A & 7 & Pulsed & $\mathrm{N} / \mathrm{A}$ & 270 & 16 & $\begin{array}{l}1.11 \\
\times \\
0.95 \\
\times \\
2.18\end{array}$ & $\begin{array}{l}\text { Seated / } \\
\text { Standing / } \\
\text { Wheelchair }\end{array}$ & $\begin{array}{l}5 \times 5 ; 8.5 \times \\
5 ; 8.5 \times 9.3 \\
12 \times 10\end{array}$ & 67 & No & MAR \\
\hline Acteon Group & X-Mind Trium TLD & $\begin{array}{l}\text { CsI / } \\
\text { CMOS }\end{array}$ & $80-90$ & $4-12$ & $\begin{array}{l}7.0 \\
\text { mmAl }\end{array}$ & 0.5 & $0.075-0.150$ & $18-27$ & $4-12$ & Pulsed & 29 & 360 & N/A & $\begin{array}{l}1.50 \\
\times \\
1.10 \\
\times \\
2.36\end{array}$ & $\begin{array}{l}\text { Seated / } \\
\text { Standing / } \\
\text { Wheelchair }\end{array}$ & $\begin{array}{l}4 \times 4 ; 6 \times 6 \\
8 \times 8 ; 11 \times 8\end{array}$ & 185 & No & MAR \\
\hline Air Techniques & Provecta 3D Prime & $\begin{array}{l}\text { CsI / } \\
\text { CMOS }\end{array}$ & $50-99$ & $4-16$ & $\begin{array}{l}5.8 \\
\text { mmAl }\end{array}$ & 0.5 & $0.080-0.200$ & $2-18$ & $0.5-20$ & Pulsed & N/A & $180-540$ & 14 & $\begin{array}{l}1.55 \\
\times \\
1.21 \\
\times \\
2.25\end{array}$ & $\begin{array}{l}\text { Seated / } \\
\text { Standing / } \\
\text { Wheelchair }\end{array}$ & $\begin{array}{l}5 \times 5 ; 13 \times \\
7 ; 13 \times 8.5\end{array}$ & 180 & No & MAR \\
\hline Asahi Roentgen & Solio $\mathrm{X}$ & $\begin{array}{l}\text { CsI / } \\
\text { aSi }\end{array}$ & $60-85$ & $2-8$ & $\begin{array}{l}2.5 \\
\text { mmAl }\end{array}$ & 0.6 & 0.100 & $\mathrm{~N} / \mathrm{A}$ & $\begin{array}{l}6-12 \\
\left(180^{\circ} /\right. \\
\left.360^{\circ}\right)\end{array}$ & $\mathrm{N} / \mathrm{A}$ & N/A & $\begin{array}{l}180 / \\
270 / \\
360\end{array}$ & $\mathrm{~N} / \mathrm{A}$ & $\begin{array}{l}1.29 \\
\times \\
0.99 \\
\times \\
2.28\end{array}$ & $\begin{array}{l}\text { Seated / } \\
\text { Standing / } \\
\text { Wheelchair }\end{array}$ & $\begin{array}{l}5.1 \times 5.5 ; 9 \\
\times 9.1\end{array}$ & 130 & No & MAR \\
\hline Asahi Roentgen & Auge Solio Z & $\begin{array}{l}\text { CsI / } \\
\text { aSi }\end{array}$ & $60-100$ & $2-12$ & $\begin{array}{l}2.5 \\
\text { mmAl }\end{array}$ & 0.6 & $0.100-0.315$ & $8.5-17$ & $8.5-17$ & Continuous & $\mathrm{N} / \mathrm{A}$ & $\begin{array}{l}180 / \\
270 / \\
360\end{array}$ & 12 & $\begin{array}{l}1.37 \\
\times \\
1.16 \\
\times \\
2.29\end{array}$ & $\begin{array}{l}\text { Seated / } \\
\text { Standing / } \\
\text { Wheelchair }\end{array}$ & $\begin{array}{l}5.1 \times 5.5 \\
9.7 \times 10 \\
16.1 \times 10\end{array}$ & 200 & No & $\begin{array}{l}\text { MAR, scout } \\
\text { image }\end{array}$ \\
\hline Carestream & CS 8100 3D & $\begin{array}{l}\text { CsI / } \\
\text { CMOS }\end{array}$ & $60-90$ & $2-15$ & $\begin{array}{l}2.5 \\
\text { mmAl }\end{array}$ & 0.6 & $0.075-0.400$ & $3-15$ & $3-15$ & Continuous & $<120$ & 220 & 14 & $\begin{array}{l}1.20 \\
\times \\
1.40 \\
\times \\
2.40\end{array}$ & $\begin{array}{l}\text { Seated / } \\
\text { Standing / } \\
\text { Wheelchair }\end{array}$ & $\begin{array}{l}4 \times 4 ; 5 \times 5 \\
8 \times 5 ; 8 \times 8 \\
8 \times 9\end{array}$ & 95 & No & MAR \\
\hline Carestream & CS 8200 3D & $\begin{array}{l}\text { CsI / } \\
\text { CMOS }\end{array}$ & $60-90$ & $2-15$ & $\begin{array}{l}2.5 \\
\text { mmAl }\end{array}$ & 0.6 & $0.075-0.400$ & $3-15$ & $3-15$ & Continuous & $<120$ & 220 & 14 & $\mathrm{~N} / \mathrm{A}$ & $\begin{array}{l}\text { Seated / } \\
\text { Standing / } \\
\text { Wheelchair }\end{array}$ & $\begin{array}{l}4 \times 4 ; 5 \times 5 \\
8 \times 5 ; 8 \times 8 \\
12 \times 10\end{array}$ & 95 & No & MAR \\
\hline Carestream & CS 9300 & & $60-90$ & $2-15$ & & 0.7 & $0.090-0.500$ & $12-28$ & $6.2-20$ & & $32-55$ & & 16 & & & & 160 & $\begin{array}{l}\text { No } \\
\text { (contir }\end{array}$ & inued on next page) \\
\hline
\end{tabular}




\begin{tabular}{|c|c|c|c|c|c|c|c|c|c|c|c|c|c|c|c|c|c|c|c|}
\hline 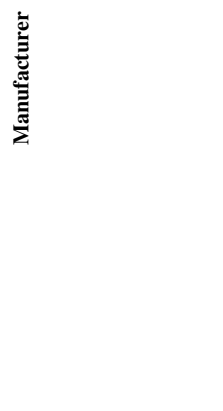 & 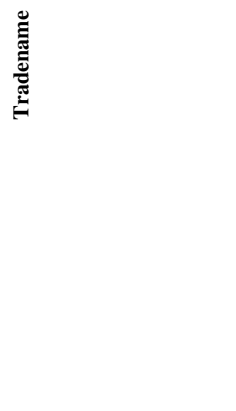 & 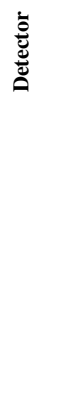 & 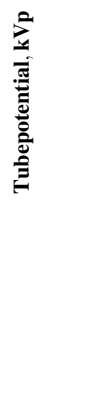 & 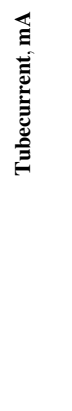 & 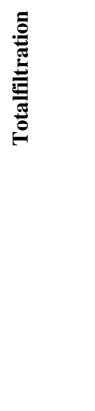 & 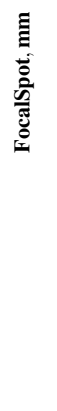 & 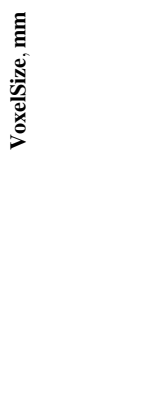 & 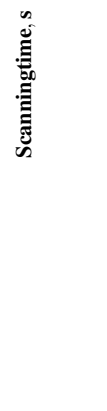 & 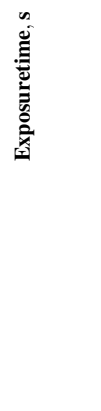 & : & 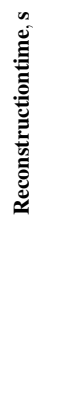 & 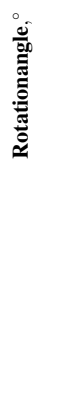 & 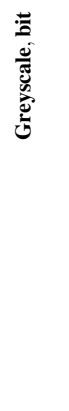 & 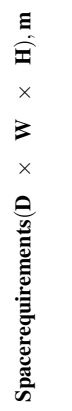 & 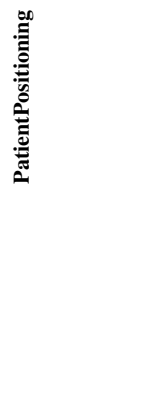 & 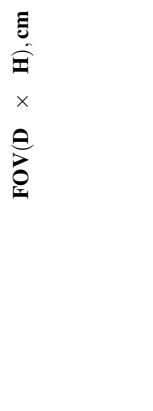 & 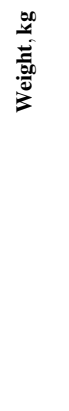 & 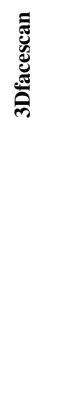 & 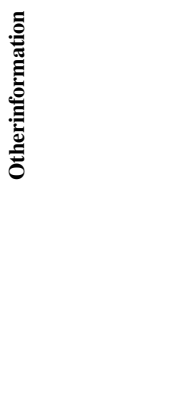 \\
\hline & & $\begin{array}{l}\text { CsI / } \\
\text { aSi }\end{array}$ & & & $\begin{array}{l}2.5 \\
\text { mmAl }\end{array}$ & & & & & $\begin{array}{l}\text { Continuous } \\
\text { / pulsed }\end{array}$ & & $\begin{array}{l}190 / \\
360\end{array}$ & & $\begin{array}{l}1.60 \\
\times \\
1.16 \\
\times \\
2.38\end{array}$ & $\begin{array}{l}\text { Seated / } \\
\text { Standing / } \\
\text { Wheelchair }\end{array}$ & $\begin{array}{l}5 \times 5 ; 8 \times 8 \\
10 \times 5 ; 10 \times \\
10 ; 17 \times 6 \\
17 \times 11 ; 17 \\
\times 13.5\end{array}$ & & & \\
\hline Carestream & CS 9600 & $\begin{array}{l}\text { CsI / } \\
\text { CMOS }\end{array}$ & $\begin{array}{l}60-90 \\
(60-120 \\
\text { option } \\
\text { CBCT } \\
90-120)\end{array}$ & $2-15$ & $\begin{array}{l}2.5 \\
\mathrm{mmAl} \\
+0.15 \\
/ 0.7 \\
\mathrm{mmCu}\end{array}$ & $\begin{array}{l}0.3 \\
/ \\
0.7\end{array}$ & $0.075-0.400$ & $5.5-40$ & $5.5-40$ & Continuous & N/A & 360 & 14 & $\begin{array}{l}1.67 \\
\times \\
1.28 \\
\times \\
2.53\end{array}$ & $\begin{array}{l}\text { Seated / } \\
\text { Standing / } \\
\text { Wheelchair }\end{array}$ & $\begin{array}{l}4 \times 4 ; 5 \times 5 \\
5 \times 8 ; 6 \times 6 \\
8 \times 5 ; 8 \times 8 \\
10 \times 5 ; 10 \times \\
10 ; 12 \times 5 \\
12 \times 10 ; 16 \\
\times 6 ; 16 \times \\
10 ; 16 \times 12 \\
16 \times 17\end{array}$ & 210 & Yes & $\begin{array}{l}\text { MAR, scout } \\
\text { image }\end{array}$ \\
\hline Carestream & 9500 & $\begin{array}{l}\text { CsI / } \\
\text { aSi }\end{array}$ & $60-90$ & $2-15$ & $\begin{array}{l}2.5 \\
\text { mmAl }\end{array}$ & 0.7 & $0.200-0.300$ & 24 & 10.8 & Pulsed & $<140$ & 360 & 14 & $\begin{array}{l}1.73 \\
\times \\
1.64 \\
\times \\
2.40\end{array}$ & $\begin{array}{l}\text { Seated / } \\
\text { Standing / } \\
\text { Wheelchair }\end{array}$ & $\begin{array}{l}9 \times 15 ; 18.4 \\
\times 20.6\end{array}$ & 176 & No & \\
\hline Cefla Dental Group & MyRay Hyperion X5 & $\begin{array}{l}\text { CsI / } \\
\text { aSi }\end{array}$ & 90 & $4-15$ & $\begin{array}{l}6 \\
\mathrm{mmAl}\end{array}$ & 0.6 & $0.080-0.160$ & $6.4-16.8$ & $1.6-4.4$ & Pulsed & $0-15$ & N/A & 16 & $\begin{array}{l}1.03 \\
\times \\
0.87 \\
\times \\
2.25\end{array}$ & $\begin{array}{l}\text { Seated / } \\
\text { Standing / } \\
\text { Wheelchair }\end{array}$ & $\begin{array}{l}6 \times 6 ; 6 \times 7 \\
8 \times 6 ; 8 \times 7 \\
10 \times 6 ; 10 \times \\
7 ; 10 \times 10\end{array}$ & 99 & No & $\begin{array}{l}\text { MAR, scout } \\
\text { image }\end{array}$ \\
\hline Cefla Dental Group & $\begin{array}{l}\text { MyRay Hyperion X9 } \\
\text { Pro }\end{array}$ & $\begin{array}{l}\text { CsI / } \\
\text { aSi }\end{array}$ & 90 & $2-16$ & $\begin{array}{l}6.5 \\
\text { mmAl }\end{array}$ & 0.5 & $0.068-0.150$ & $3.6-14.4$ & $1.6-8.0$ & Pulsed & $0-15$ & $\begin{array}{l}180 / \\
360\end{array}$ & 16 & $\begin{array}{l}1.14 \\
\times \\
1.39 \\
\times \\
2.45\end{array}$ & $\begin{array}{l}\text { Seated / } \\
\text { Standing / } \\
\text { Wheelchair }\end{array}$ & $\begin{array}{l}4 \times 4 ; 6 \times 6 \\
7 \times 6 ; 8 \times 6 \\
8 \times 8 ; 10 \times \\
6 ; 10 \times 8 ; 10 \\
\times 10 ; 13 \times \\
8 ; 13 \times 10 \\
13 \times 16\end{array}$ & 155 & No & $\begin{array}{l}\text { MAR, scout } \\
\text { image, real-time } \\
\text { mA-modulation }\end{array}$ \\
\hline Dabi Atlante & Eagle 3D & $\begin{array}{l}\text { CsI / } \\
\text { CMOS }\end{array}$ & $60-85$ & $4-8$ & $\begin{array}{l}2.5 \\
\text { mmAl }\end{array}$ & 0.5 & $0.080-0.500$ & N/A & $7-32$ & Pulsed & $22-97$ & N/A & N/A & $\begin{array}{l}1.27 \\
\times \\
1.12 \\
\times \\
2.44\end{array}$ & $\begin{array}{l}\text { Seated / } \\
\text { Standing / } \\
\text { Wheelchair }\end{array}$ & $\begin{array}{l}5 \times 5 ; 6 \times 8 \\
8 \times 8 ; 8 \times \\
12 ; 8 \times 16 \\
13 \times 16 ; 18 \\
\times 16 ; 23 \times \\
16\end{array}$ & 115 & No & $\begin{array}{l}\text { MAR, scout } \\
\text { image, motion } \\
\text { correction }\end{array}$ \\
\hline Dentium & Rainbow CT & $\begin{array}{l}\text { CsI / } \\
\text { CMOS }\end{array}$ & $60-100$ & $4-12$ & $\mathrm{~N} / \mathrm{A}$ & 0.5 & $0.200-0.300$ & 19 & $\mathrm{~N} / \mathrm{A}$ & $\mathrm{N} / \mathrm{A}$ & $<60$ & $\mathrm{~N} / \mathrm{A}$ & N/A & N/A & $\begin{array}{l}\text { Seated / } \\
\text { Standing / } \\
\text { Wheelchair }\end{array}$ & $\begin{array}{l}5 \times 5 ; 16 \times \\
10 ; 16 \times 18\end{array}$ & $\mathrm{~N} / \mathrm{A}$ & No & \\
\hline Dentsply Sirona & Orthophos SL 3D & $\begin{array}{l}\text { CsI / } \\
\text { aSi }\end{array}$ & $60-90$ & $3-16$ & $\begin{array}{l}2.5 \\
\text { mmAl } \\
+0.3\end{array}$ & 0.5 & $0.080-0.220$ & $2.2-14.4$ & $\mathrm{~N} / \mathrm{A}$ & Pulsed & N/A & $\mathrm{N} / \mathrm{A}$ & N/A & $\begin{array}{l}1.37 \\
\times \\
1.04\end{array}$ & $\begin{array}{l}\text { Seated / } \\
\text { Standing / } \\
\text { Wheelchair }\end{array}$ & $\begin{array}{l}5 \times 5.5 ; 8 \times \\
8 ; 11 \times 10\end{array}$ & N/A & No & MAR \\
\hline
\end{tabular}




\begin{tabular}{|c|c|c|c|c|c|c|c|c|c|c|c|c|c|c|c|c|c|c|c|}
\hline 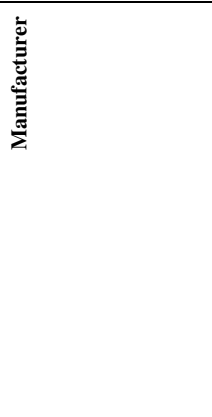 & 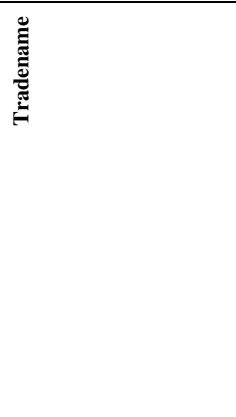 & 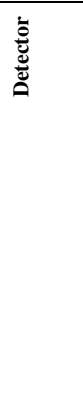 & 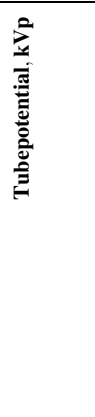 & 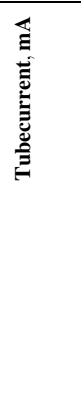 & 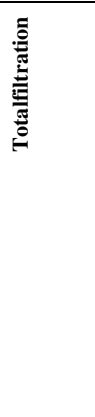 & 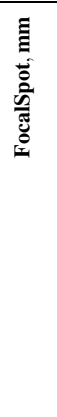 & 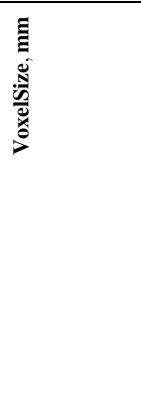 & 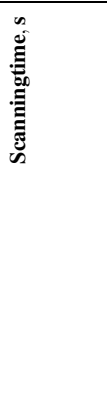 & 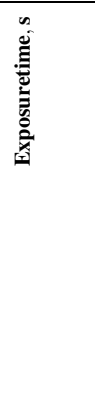 & 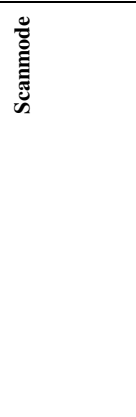 & 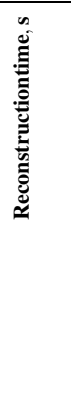 & 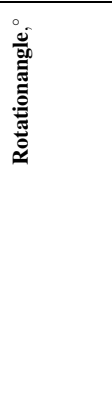 & 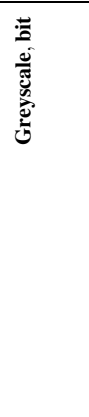 & 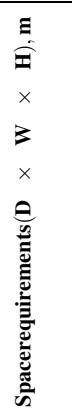 & 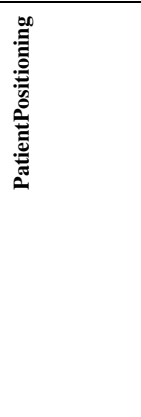 & 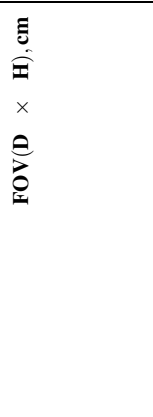 & 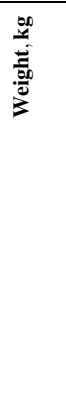 & 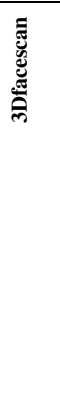 & 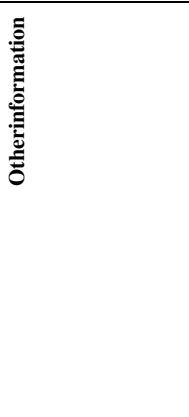 \\
\hline Dentsply Sirona & Axeos & $\begin{array}{l}\text { CsI / } \\
\text { aSi }\end{array}$ & 85 & $3-16$ & $\begin{array}{l}/ 1.0 \\
\mathrm{mmCu} \\
2.5 \\
\mathrm{mmAl} \\
+0.3 \\
/ 0.5 / \\
1.0 \\
\mathrm{mmCu}\end{array}$ & 0.5 & $0.080-0.220$ & $2.2-14.4$ & $\mathrm{~N} / \mathrm{A}$ & Pulsed & N/A & $\mathrm{N} / \mathrm{A}$ & $\mathrm{N} / \mathrm{A}$ & $\begin{array}{l}\times \\
2.25 \\
1.37 \\
\times \\
1.04 \\
\times \\
2.25\end{array}$ & $\begin{array}{l}\text { Seated / } \\
\text { Standing / } \\
\text { Wheelchair }\end{array}$ & $\begin{array}{l}5 \times 5.5 ; 8 \times \\
8 ; 11 \times 10 \\
17 \times 13\end{array}$ & 191 & No & MAR \\
\hline Dürr Dental & VistaVox S & $\begin{array}{l}\text { CsI / } \\
\text { CMOS }\end{array}$ & $50-99$ & $4-16$ & $\begin{array}{l}5.8 \\
\mathrm{mmAl}\end{array}$ & 0.5 & $0.080-0.200$ & $2-18$ & $0.5-20$ & Pulsed & $\mathrm{N} / \mathrm{A}$ & $180-540$ & 14 & $\begin{array}{l}1.55 \\
\times \\
1.21 \\
\times \\
2.21\end{array}$ & $\begin{array}{l}\text { Seated / } \\
\text { Standing / } \\
\text { Wheelchair }\end{array}$ & $\begin{array}{l}5 \times 5 ; 13 \times \\
7 ; 13 \times 8.5\end{array}$ & 180 & No & \\
\hline Genoray & Papaya 3D Premium & N/A & $60-90$ & 4-12 & N/A & 0.5 & $0.075-0.400$ & $7.7-14.5$ & $\mathrm{~N} / \mathrm{A}$ & N/A & N/A & $\begin{array}{l}180 / \\
360\end{array}$ & $\mathrm{~N} / \mathrm{A}$ & $\begin{array}{l}1.20 \\
\times \\
1.13 \\
\times \\
2.38\end{array}$ & $\begin{array}{l}\text { Seated / } \\
\text { Standing / } \\
\text { Wheelchair }\end{array}$ & $\begin{array}{l}3.5 \times 4 ; 4 \times \\
5 ; 7 \times 7 ; 8 \times \\
8 ; 14 \times 8 ; 14 \\
\times 14 ; 23 \times \\
24(19 \\
\text { programs } \\
\text { available) }\end{array}$ & 160 & No & $\begin{array}{l}\text { Freely adjustable } \\
\text { FOV }\end{array}$ \\
\hline HDX Will & DENTRI $\alpha$ & $\begin{array}{l}\text { CsI / } \\
\text { CMOS } \\
\text { or CsI } \\
/ \text { aSi }\end{array}$ & $80-110$ & $4-10$ & $\begin{array}{l}2.5 \\
\mathrm{mmAl}\end{array}$ & 0.5 & $0.100-0.300$ & $\mathrm{~N} / \mathrm{A}$ & $8-36$ & Pulsed & $<60$ & 360 & $14-16$ & $\begin{array}{l}1.36 \\
\times \\
1.20 \\
\times \\
2.46\end{array}$ & $\begin{array}{l}\text { Seated / } \\
\text { Standing / } \\
\text { Wheelchair }\end{array}$ & $\begin{array}{l}16 \times 8 ; 16 \times \\
14.5 \text { (freely } \\
\text { adjustable } \\
\text { FOV) }\end{array}$ & 243 & No & $\begin{array}{l}\text { Freely adjustable } \\
\text { FOV, scout } \\
\text { image, MAR }\end{array}$ \\
\hline HDX Will & Eco-X & $\begin{array}{l}\text { CsI / } \\
\text { aSi }\end{array}$ & $60-90$ & $4-10$ & $\begin{array}{l}2.5 \\
\text { mmAl }\end{array}$ & 0.5 & $0.200-0.300$ & $\mathrm{~N} / \mathrm{A}$ & $8-24$ & Pulsed & N/A & 360 & 14 & $\begin{array}{l}1.23 \\
\times \\
0.98 \\
\times \\
2.31\end{array}$ & $\begin{array}{l}\text { Seated / } \\
\text { Standing / } \\
\text { Wheelchair }\end{array}$ & $\begin{array}{l}10 \times 8 ; 12 \times \\
10 ; 16 \times 9\end{array}$ & $\mathrm{~N} / \mathrm{A}$ & No & $\begin{array}{l}\text { AI-MAR, mA- } \\
\text { modulation }\end{array}$ \\
\hline HDX Will & Q-Face & $\begin{array}{l}\text { CsI / } \\
\text { CMOS }\end{array}$ & $60-90$ & $4-10$ & $\begin{array}{l}2.5 \\
\text { mmAl }\end{array}$ & 0.5 & $0.100-0.300$ & $8-36$ & $\mathrm{~N} / \mathrm{A}$ & Pulsed & $<60$ & 360 & 14 & $\begin{array}{l}1.39 \\
\times \\
1.53 \\
\times \\
2.36\end{array}$ & $\begin{array}{l}\text { Seated / } \\
\text { Standing / } \\
\text { Wheelchair }\end{array}$ & $\begin{array}{l}5 \times 16 ; 8 \times \\
16 ; 9 \times 16 \\
14.5 \times 16 \\
16 \times 16 ; 16 \\
\times 21\end{array}$ & 305 & Yes & MAR \\
\hline ImageWorks Corp. & Panoura X-era & $\begin{array}{l}\text { CsI / } \\
\text { CMOS }\end{array}$ & $70-90$ & $2-4$ & $\begin{array}{l}2.5 \\
\text { mmAl }\end{array}$ & 0.2 & $0.090-0.230$ & $\mathrm{~N} / \mathrm{A}$ & $12-20$ & $\mathrm{~N} / \mathrm{A}$ & $\mathrm{N} / \mathrm{A}$ & 360 & 16 & $\begin{array}{l}1.37 \\
\times \\
1.07 \\
\times \\
2.31\end{array}$ & $\begin{array}{l}\text { Seated / } \\
\text { Standing / } \\
\text { Wheelchair }\end{array}$ & $\begin{array}{l}4.4 \times 6.4 ; 8 \\
\times 7.9 ; 11 \times \\
7.9 ; 15.6 \times \\
7.9\end{array}$ & 210 & No & \\
\hline Imtec & Iluma LFOV & $\begin{array}{l}\text { CsI / } \\
\text { aSi }\end{array}$ & 120 & $1-3.8$ & $\mathrm{~N} / \mathrm{A}$ & 0.3 & $0.090-0.400$ & $7.8-40$ & $7.8-40$ & Continuous & 120 & $\begin{array}{l}190 / \\
360\end{array}$ & 16 & $\begin{array}{l}1.68 \\
\times \\
1.96\end{array}$ & Seated & $\begin{array}{l}\text { up to } 21.1 \times \\
14.2\end{array}$ & 350 & No & \\
\hline
\end{tabular}




\begin{tabular}{|c|c|c|c|c|c|c|c|c|c|c|c|c|c|c|c|c|c|c|c|}
\hline 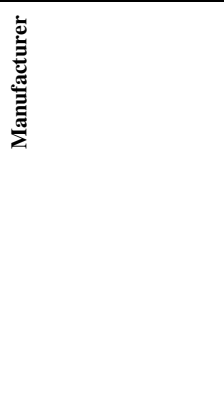 & 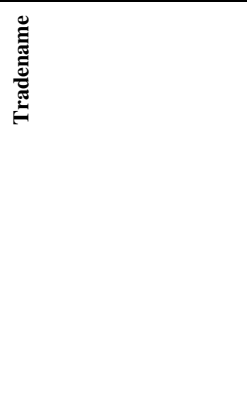 & 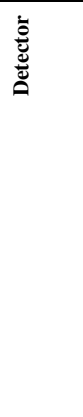 & 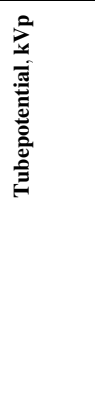 & 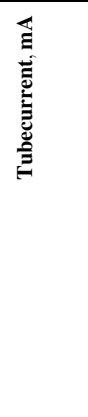 & 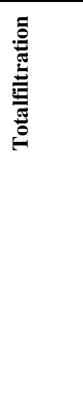 & 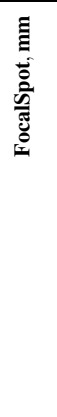 & 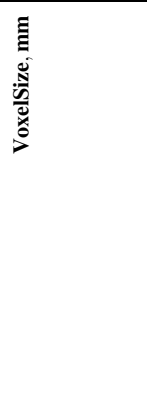 & 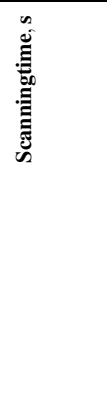 & 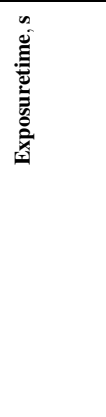 & 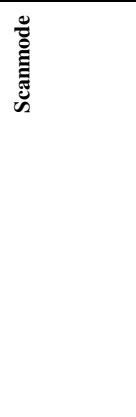 & 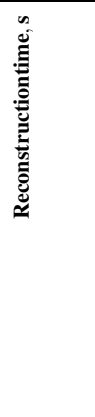 & 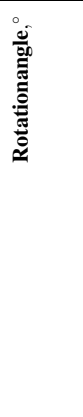 & 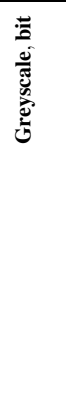 & 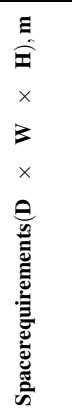 & 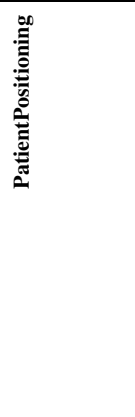 & 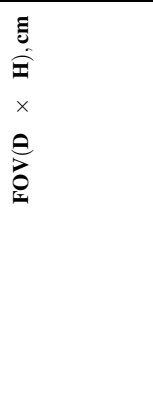 & 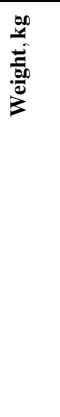 & 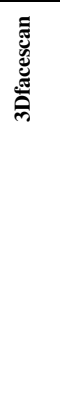 & 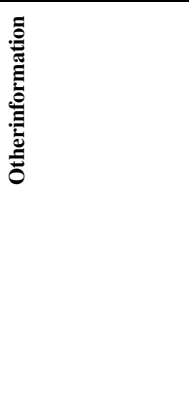 \\
\hline Imtec & Iluma SFOV & $\begin{array}{l}\text { CsI / } \\
\text { aSi }\end{array}$ & 120 & $1-3.8$ & N/A & 0.3 & $0.090-0.400$ & $7.8-40$ & $7.8-40$ & Continuous & 120 & $\begin{array}{l}190 / \\
360\end{array}$ & 16 & $\begin{array}{l}\times \\
2.16 \\
1.68 \\
\times \\
1.96 \\
\times \\
2.16\end{array}$ & Seated & $\begin{array}{l}\text { up to } 10.8 \times \\
9.6\end{array}$ & 350 & No & \\
\hline Instrumentarium & $\begin{array}{l}\text { Orthopantomograph } \\
\text { OP300 }\end{array}$ & $\begin{array}{l}\text { CsI / } \\
\text { CMOS }\end{array}$ & $57-90$ & $4-16$ & $\begin{array}{l}3.2 \\
\text { mmAl }\end{array}$ & 0.5 & $0.085-0.300$ & $10-20$ & $2.3-12.5$ & Pulsed & $<30$ & 360 & 16 & $\begin{array}{l}1.39 \\
\times \\
0.97 \\
\times \\
2.41\end{array}$ & $\begin{array}{l}\text { Seated / } \\
\text { Standing / } \\
\text { Wheelchair }\end{array}$ & $6 \times 4 ; 6 \times 8$ & 200 & No & $\begin{array}{l}\text { MAR, scout } \\
\text { image }\end{array}$ \\
\hline $\begin{array}{c}\text { Instrumentarium/ } \\
\text { PaloDEx / Kavo }\end{array}$ & $\begin{array}{l}\text { Orthopantomograph } \\
\text { OP 3D }\end{array}$ & $\begin{array}{l}\text { CsI / } \\
\text { CMOS }\end{array}$ & $\begin{array}{l}60-95 \\
\text { (CBCT } \\
95)\end{array}$ & $2-16$ & $\begin{array}{l}3.2 \\
\mathrm{mmAl}\end{array}$ & 0.5 & $0.080-0.400$ & $27-45$ & $1.7-20$ & Pulsed & $<30$ & 360 & 16 & $\begin{array}{l}1.10 \\
\times \\
0.77 \\
\times \\
2.44\end{array}$ & $\begin{array}{l}\text { Seated / } \\
\text { Standing / } \\
\text { Wheelchair }\end{array}$ & $\begin{array}{l}5 \times 5 ; 6 \times 9 \\
6 \times 11 ; 9 \times \\
14\end{array}$ & 120 & No & $\begin{array}{l}\text { MAR, scout } \\
\text { image, FOV } \\
\text { adjustable in } \\
\text { height direction } \\
(0.5 \mathrm{~cm} \text { steps } \\
\text { between } 5 \text { and } 9 \\
\mathrm{~cm})\end{array}$ \\
\hline $\begin{array}{l}\text { Instrumentarium / } \\
\text { PaloDEx / Kavo }\end{array}$ & $\begin{array}{l}\text { Orthopantomograph } \\
\text { OP 3D Pro }\end{array}$ & $\begin{array}{l}\text { CsI / } \\
\text { CMOS }\end{array}$ & $\begin{array}{l}57-90 \\
\text { (CBCT } \\
90)\end{array}$ & $3.2-16$ & $\begin{array}{l}3.2 \\
\text { mmAl }\end{array}$ & 0.5 & $0.085-0.420$ & $11-42$ & $1.2-12.6$ & Pulsed & $<30$ & 360 & 16 & $\begin{array}{l}1.13 \\
\times \\
0.83 \\
\times \\
2.41\end{array}$ & $\begin{array}{l}\text { Seated / } \\
\text { Standing / } \\
\text { Wheelchair }\end{array}$ & $\begin{array}{l}5 \times 5 ; 6 \times 8 \\
8 \times 8 ; 8 \times \\
15 ; 13 \times 15\end{array}$ & 200 & No & $\begin{array}{l}\text { MAR, scout } \\
\text { image. FOV } \\
\text { freely positioned }\end{array}$ \\
\hline $\begin{array}{c}\text { Instrumentarium/ } \\
\text { PaloDEx / Kavo }\end{array}$ & $\begin{array}{l}\text { Orthopantomograph } \\
\text { OP 3D Vision = } \mathrm{i} \text {-CAT } \\
\text { V17 }\end{array}$ & $\begin{array}{l}\text { CsI / } \\
\text { aSi }\end{array}$ & $90-120$ & $3-8$ & $\begin{array}{l}10 \\
\mathrm{mmAl}\end{array}$ & 0.5 & $0.125-0.400$ & $4.8-26.9$ & $2-7.4$ & Pulsed & $<30$ & 360 & 16 & $\begin{array}{l}1.34 \\
\times \\
1.22 \\
\times \\
1.82\end{array}$ & $\begin{array}{l}\text { Seated / } \\
\text { wheelchair }\end{array}$ & $\begin{array}{l}5 \times 8 ; 8 \times 8 \\
4 \times 16 ; 6 \times \\
16 ; 8 \times 16 \\
10 \times 16 ; 11 \\
\times 16 ; 13 \times \\
16 ; 17 \times 23\end{array}$ & 231 & Yes & $\begin{array}{l}\text { scout image } \\
\text { (option) }\end{array}$ \\
\hline LargeV Instrument & Smart3D-X & $\begin{array}{l}\text { CsI / } \\
\text { CMOS }\end{array}$ & $60-100$ & $2-10$ & N/A & 0.5 & $0.050-0.250$ & $\mathrm{~N} / \mathrm{A}$ & $9.5-18.5$ & Pulsed & $<60$ & 360 & N/A & $\begin{array}{l}1.51 \\
\times \\
1.79 \\
\times \\
2.36\end{array}$ & $\begin{array}{l}\text { Seated / } \\
\text { Standing / } \\
\text { Wheelchair }\end{array}$ & $\begin{array}{l}5 \times 8 ; 8 \times 8 \\
12 \times 8 ; 15 \times \\
9 ; 16 \times 10\end{array}$ & 250 & No & $\begin{array}{l}\text { deep-learning } \\
\text { based } \\
\text { reconstruction } \\
\text { algorithm, MAR, } \\
\text { panoramic image } \\
\text { can be } \\
\text { reconstructed } \\
\text { from CBCT } \\
\text { image dataset }\end{array}$ \\
\hline Morita & $\begin{array}{l}\text { Veraviewepocs 3D } \\
\text { R100 }\end{array}$ & $\begin{array}{l}\text { CsI / } \\
\text { CMOS }\end{array}$ & $75-90$ & $1-10$ & $\begin{array}{l}2.5 \\
\mathrm{mmAl} \\
+0.2 \\
\mathrm{mmCu}\end{array}$ & 0.5 & 0.125 & 9.4 & 9.4 & Continuous & $60-240$ & $\begin{array}{l}180 / \\
360\end{array}$ & 14 & $\begin{array}{l}1.30 \\
\times \\
1.02 \\
\times \\
2.36\end{array}$ & $\begin{array}{l}\text { Standing / } \\
\text { Wheelchair }\end{array}$ & $\begin{array}{l}4 \times 4 ; 4 \times 8 \\
8 \times 8\end{array}$ & 190 & No & scout image \\
\hline
\end{tabular}




\begin{tabular}{|c|c|c|c|c|c|c|c|c|c|c|c|c|c|c|c|c|c|c|c|}
\hline 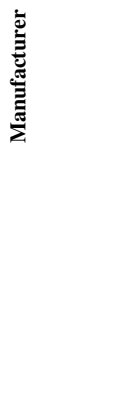 & 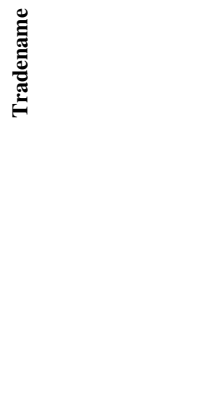 & 莺 & 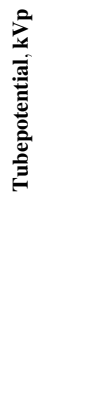 & 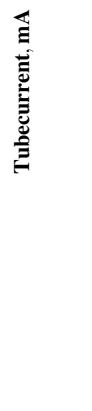 & 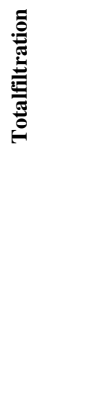 & 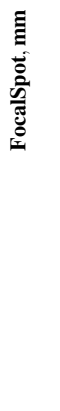 & 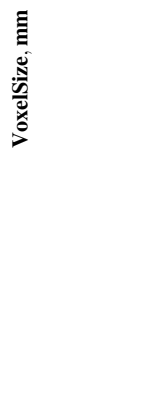 & 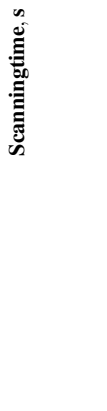 & 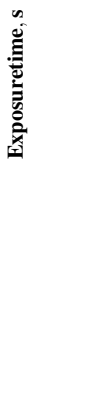 & 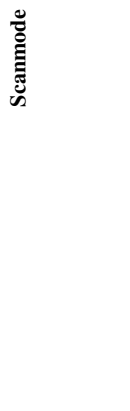 & 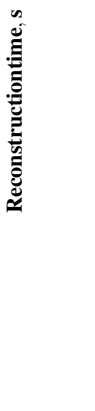 & 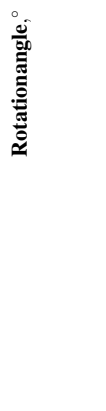 & 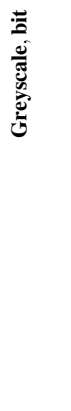 & 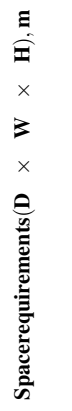 & 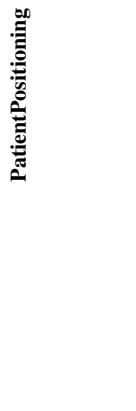 & $\begin{array}{l}5 \\
\vdots \\
\vdots \\
\times \\
e \\
0 \\
0\end{array}$ & 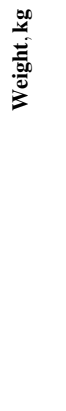 & 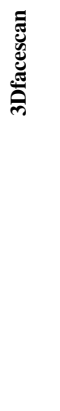 & 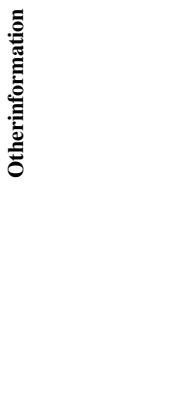 \\
\hline Morita & 3D Accuitomo 170 & $\begin{array}{l}\text { CsI / } \\
\text { aSi }\end{array}$ & $60-90$ & $1-10$ & $\begin{array}{l}3.1 \\
\mathrm{mmAl}\end{array}$ & 0.5 & $0.080-0.250$ & $5.4-30.8$ & $5.4-30.8$ & Continous & $20-120$ & $\begin{array}{l}180 / \\
360\end{array}$ & 14 & $\begin{array}{l}1.20 \\
\times \\
1.62 \\
\times \\
2.08\end{array}$ & Seated & $\begin{array}{l}4 \times 4 ; 6 \times 6 \\
8 \times 8 ; 10 \times \\
5 ; 10 \times 10 \\
14 \times 5 ; 14 \times \\
10 ; 17 \times 5 \\
17 \times 12\end{array}$ & 400 & No & scout image \\
\hline Morita & Veraview X800 & N/A & $60-100$ & $2-10$ & $\begin{array}{l}2.5 \\
\mathrm{mmAl} \\
+0.2 \\
\mathrm{mmCu}\end{array}$ & 0.5 & $0.080-$ & 9.4-17.9 & 9.4-17.9 & Continous & $\mathrm{N} / \mathrm{A}$ & $\begin{array}{l}180 / \\
360\end{array}$ & N/A & $\begin{array}{l}1.20 \\
\times \\
1.40 \\
\times \\
2.33\end{array}$ & $\begin{array}{l}\text { Seated / } \\
\text { Standing / } \\
\text { Wheelchair }\end{array}$ & $\begin{array}{l}4 \times 4 ; 4 \times 8 \\
8 \times 4 ; 8 \times 5 \\
8 \times 8 ; 10 \times \\
4 ; 10 \times 5 ; 10 \\
\times 8 ; 15 \times 5 \\
15 \times 7.5 ; 15 \\
\times 14\end{array}$ & 185 & No & scout image \\
\hline Owandy & I-Max Touch 3D & $\begin{array}{l}\text { CsI / } \\
\text { aSi }\end{array}$ & $60-86$ & $6-10$ & $\begin{array}{l}2.5 \\
\mathrm{mmAl}\end{array}$ & 0.5 & 0.092 & 20 & 8 & Pulsed & 120 & $\begin{array}{l}180 \\
(\mathrm{TMJ}) / \\
200\end{array}$ & 14 & $\begin{array}{l}1.27 \\
\times \\
1.14 \\
\times \\
2.45\end{array}$ & $\begin{array}{l}\text { standing/ } \\
\text { seated/ } \\
\text { wheelchair }\end{array}$ & $\begin{array}{l}4 \times 9 ; 5 \times 9 \\
9 \times 8\end{array}$ & 161 & No & \\
\hline Owandy & I-Max 3D & $\begin{array}{l}\text { CSI / } \\
\text { CMOS }\end{array}$ & $60-86$ & $2-12.5$ & $\begin{array}{l}2.5 \\
\mathrm{mmAl}\end{array}$ & 0.5 & $0.088-0.175$ & 21.2 & $6.2-7$ & Pulsed & $\mathrm{N} / \mathrm{A}$ & 270 & 16 & $\begin{array}{l}1.27 \\
\times \\
0.95 \\
\times \\
2.23\end{array}$ & $\begin{array}{l}\text { Seated / } \\
\text { Standing / } \\
\text { Wheelchair }\end{array}$ & $\begin{array}{l}5 \times 5 ; 8.5 \times \\
5 ; 8.5 \times 9.3 \\
11.6 \times 10.2\end{array}$ & 67 & No & MAR \\
\hline Planmeca & ProMax 3D s & $\begin{array}{l}\text { CsI / } \\
\text { aSi }\end{array}$ & $60-90$ & $1-14$ & $\begin{array}{l}2.5 \\
\mathrm{mmAl} \\
+0.5 \\
\mathrm{mmCu}\end{array}$ & 0.5 & $0.075-0.400$ & $\mathrm{~N} / \mathrm{A}$ & $3-36$ & Pulsed & $2-25$ & 200 & 16 & $\begin{array}{l}1.25 \\
\times \\
1.00 \\
\times \\
2.34\end{array}$ & $\begin{array}{l}\text { Seated / } \\
\text { Standing / } \\
\text { Wheelchair }\end{array}$ & $\begin{array}{l}4.2 \times 4.2 ; \\
4.2 \times 6.8 ; 5 \\
\times 5 ; 5 \times 8 ; \\
6.8 \times 4.2 ; 8 \\
\times 4 ; 8 \times 5 \\
\text { (+double } \\
\text { and triple } \\
\text { scans) }\end{array}$ & 119 & Yes & $\begin{array}{l}\text { Advanced } \\
\text { stitching with up } \\
\text { to three } \\
\text { horizontal } \\
\text { volumes and two } \\
\text { vertical volumes, } \\
\text { adaptive image } \\
\text { noise optimizer, } \\
\text { MAR, movement } \\
\text { correction }\end{array}$ \\
\hline Planmeca & ProMax 3D Classic & $\begin{array}{l}\text { CsI / } \\
\text { aSi }\end{array}$ & $60-90$ & $1-14$ & $\begin{array}{l}2.5 \\
\mathrm{mmAl} \\
+0.5 \\
\mathrm{mmCu}\end{array}$ & 0.5 & $0.075-0.400$ & $\mathrm{~N} / \mathrm{A}$ & $3-36$ & Pulsed & $2-25$ & 200 & 16 & $\begin{array}{l}1.25 \\
\times \\
1.00 \\
\times \\
2.34\end{array}$ & $\begin{array}{l}\text { Seated / } \\
\text { Standing / } \\
\text { Wheelchair }\end{array}$ & $\begin{array}{l}4.2 \times 4.2 ; \\
4.2 \times 6.8 ; 5 \\
\times 5 ; 5 \times 8 ; \\
6.8 \times 4.2 ; \\
6.8 \times 6.8 ; 8 \\
\times 4 ; 8 \times 5 ; 8 \\
\times 8 ; 11 \times 5 ; \\
11 \times 8 ; \\
\text { (+double } \\
\text { and triple } \\
\text { scans) }\end{array}$ & 119 & Yes & $\begin{array}{l}\text { Advanced } \\
\text { stitching with up } \\
\text { to three } \\
\text { horizontal } \\
\text { volumes, } \\
\text { adaptive image } \\
\text { noise optimiser, } \\
\text { MAR, movement } \\
\text { correction }\end{array}$ \\
\hline
\end{tabular}




\begin{tabular}{|c|c|c|c|c|c|c|c|c|c|c|c|c|c|}
\hline 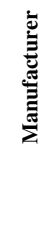 & 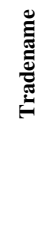 & 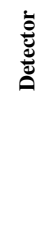 & 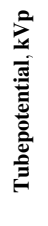 & 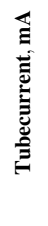 & 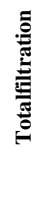 & 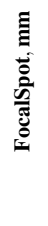 & 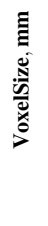 & 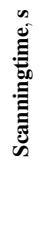 & 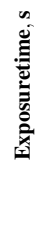 & 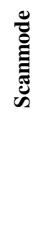 & 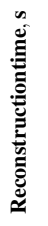 & 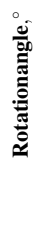 & 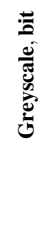 \\
\hline
\end{tabular}

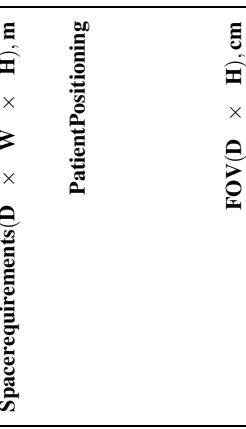

\begin{tabular}{llllll}
\hline Planmeca & ProMax 3D Plus & CsI / & $60-120$ & $1-14$ & 2.5 \\
& & aSi & & & mm \\
& & & \\
& & & \\
& & & & \\
& & & &
\end{tabular}

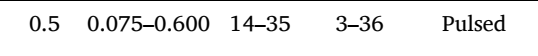

$\mathrm{mmAl}$
+0.5
$\mathrm{mmCu}$

$\begin{array}{ll}1.43 & \text { Seated / } \\ \times & \text { Standing / } \\ 1.10 & \text { Wheelchair } \\ \times & \\ 2.39 & \end{array}$

$3.4 \times 4.2$;

$3.4 \times 4.2$

$3.4 \times 6.8 ; 4$
$\times 5 ; 4 \times 8 ;$

$6.8 \times 4.2$

$6.8 \times 6.8 ; 8$

$\times 4 ; 8 \times 5 ; 8$
$\times 8 ; 8.5 \times 5 ;$

$10 \times 6 ; 8.5$

$\times 8.5 ; 10 \times$

$10 ; 16 \times 10$;

$20 \times 6 ; 20 \times$

Planmeca

ProMax 3D Mid

$\begin{array}{llll}\text { CsI / } & 60-120 & 1-14 & 2.5 \\ & & & \end{array}$

$0.5 \quad 0.075-0.600 \quad 14-35 \quad 3-36 \quad$ Pulsed

2-55 $200 / 16$

1.43 Seated /

10
$\mathrm{mmAl}$
+0.5

$\mathrm{mmCu}$

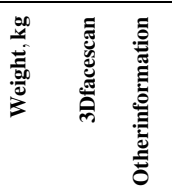

141 Yes Adaptive image noise optimiser, MAR, movemen correction

$3.4 \times 4.2$

$3.4 \times 6.8 ; 4$
$\times 5 ; 4 \times 8 ;$

$3.4 \times 6.8,4$
$\times 5 \times 4.2 ;$

141 Yes Adaptive image

$\times .8 \times 4.2 ;$
$6.8 \times 6.8 ; 8$

$\times 4 ; 8 \times 5 ; 8$

$\times 8 ; 8.5 \times 5$

$8.5 \times 8.5 ; 10$

$\times 6 ; 10 \times 8$

$10 \times 10 ; 10$

$\times 14 ; 16 \times$
$8 ; 16 \times 10$;

$6 \times 14 ; 20$

$\times 6 ; 20 \times 8$

$20 \times 10 ; 20$

$\times 14 ; 20 \times$

17

\begin{tabular}{|c|c|c|c|c|c|c|c|c|c|c|c|c|c|c|c|c|c|c|c|}
\hline Planmeca & Viso G5 & $\begin{array}{l}\text { CsI / } \\
\mathrm{aSi}\end{array}$ & $60-120$ & $1-16$ & $\begin{array}{l}2.5 \\
\mathrm{mmAl} \\
+0.2 \\
/ 0.5 \\
\mathrm{mmCu}\end{array}$ & 0.5 & $0.075-0.600$ & N/A & $1.5-36$ & Pulsed & N/A & $\begin{array}{l}210 / \\
360\end{array}$ & 16 & $\begin{array}{l}1.52 \\
\times \\
1.39 \\
\times \\
2.36\end{array}$ & $\begin{array}{l}\text { Seated / } \\
\text { Standing / } \\
\text { Wheelchair }\end{array}$ & $\begin{array}{l}3 \times 3 \text { to } 20 \\
\times 17(0.5-2 \\
\text { cm steps } \\
\text { depending } \\
\text { on the } \\
\text { program) }\end{array}$ & 165 & Yes & $\begin{array}{l}\text { Live virtual FOV } \\
\text { positioning, } \\
\text { adaptive image } \\
\text { noise optimiser, } \\
\text { MAR, movement } \\
\text { correction, 4D } \\
\text { jaw motion }\end{array}$ \\
\hline Planmeca & Viso G7 & $\begin{array}{l}\text { CsI / } \\
\mathrm{aSi}\end{array}$ & $60-120$ & $1-16$ & $\begin{array}{l}2.5 \\
\mathrm{mmAl} \\
+0.2 \\
/ 0.5 \\
\mathrm{mmCu}\end{array}$ & 0.5 & $0.075-0.600$ & N/A & $1.5-36$ & Pulsed & N/A & $\begin{array}{l}210 / \\
360\end{array}$ & 16 & $\begin{array}{l}1.52 \\
\times \\
1.39 \\
\times \\
2.36\end{array}$ & $\begin{array}{l}\text { Seated / } \\
\text { Standing / } \\
\text { Wheelchair }\end{array}$ & $\begin{array}{l}3 \times 3 \text { to } 26 \\
\times 30(0.5-2 \\
\text { cm steps } \\
\text { depending } \\
\text { on the } \\
\text { program) }\end{array}$ & 165 & Yes & $\begin{array}{l}\text { Live virtual FOV } \\
\text { positioning, } \\
\text { adaptive image } \\
\text { noise optimiser, } \\
\text { MAR, movement } \\
\text { correction, 4D } \\
\text { jaw motion }\end{array}$ \\
\hline
\end{tabular}

(continued on next page) 


\begin{tabular}{|c|c|c|c|c|c|c|c|c|c|c|c|c|c|c|c|c|c|c|c|}
\hline 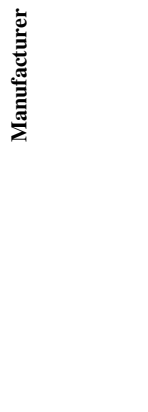 & 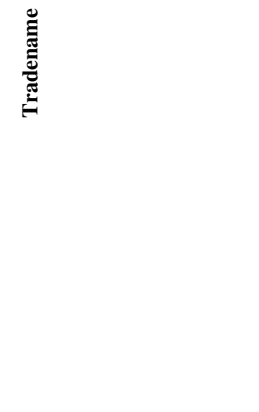 & 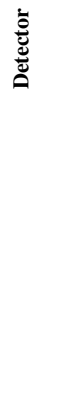 & 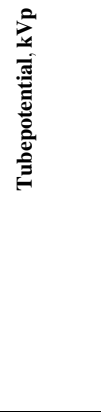 & 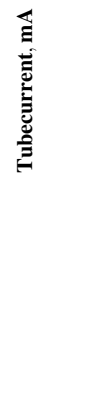 & 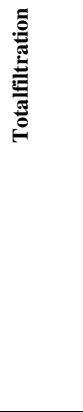 & 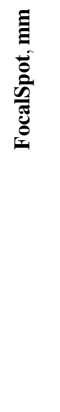 & 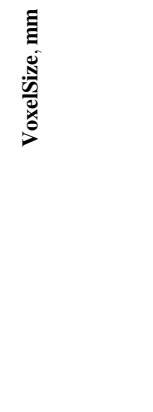 & 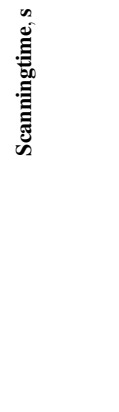 & 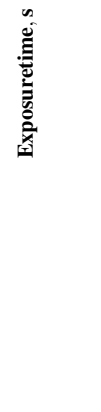 & 苋 & 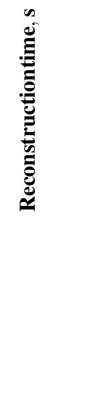 & 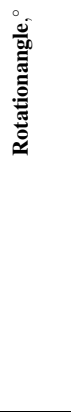 & 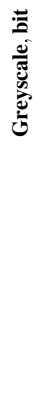 & 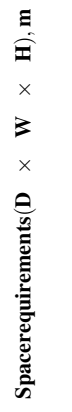 & 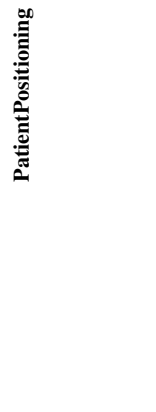 & 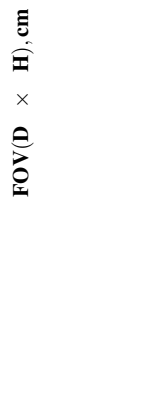 & 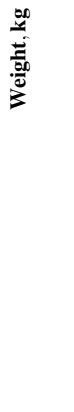 & 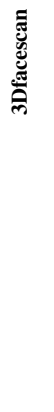 & 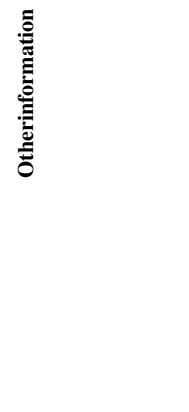 \\
\hline & $\begin{array}{l}\text { Point 3D Combi } \\
\text { 500C/s }\end{array}$ & $\begin{array}{l}\text { CsI / } \\
\text { aSi }\end{array}$ & & & & & & & & & & & & $\begin{array}{l}1.25 \\
\times \\
1.06 \\
\times \\
2.30\end{array}$ & $\begin{array}{l}\text { Seated / } \\
\text { Standing / } \\
\text { Wheelchair }\end{array}$ & $\begin{array}{l}12 \times 9 ; 14 \times \\
9\end{array}$ & & & \\
\hline PointNix & Point 800 HD 3D Plus & $\begin{array}{l}\text { CsI / } \\
\text { aSi }\end{array}$ & $50-90$ & $4-10$ & $\mathrm{~N} / \mathrm{A}$ & 0.5 & $0.183-0.427$ & 19 & N/A & $\mathrm{N} / \mathrm{A}$ & $10-40$ & 360 & 14 & $\begin{array}{l}1.25 \\
\times \\
1.06 \\
\times \\
2.30\end{array}$ & $\begin{array}{l}\text { Seated / } \\
\text { Standing / } \\
\text { Wheelchair }\end{array}$ & $\begin{array}{l}10 \times 9 ; 12 \times \\
9\end{array}$ & 150 & No & MAR \\
\hline PreXion & Excelsior & $\begin{array}{l}\text { CsI / } \\
\mathrm{aSi}\end{array}$ & $60-110$ & $1-6$ & $\mathrm{~N} / \mathrm{A}$ & 0.3 & $0.080-0.200$ & $5.2-23.6$ & $\mathrm{~N} / \mathrm{A}$ & Pulsed & $\mathrm{N} / \mathrm{A}$ & 360 & 16 & $\begin{array}{l}1.56 \\
\times \\
1.16 \\
\times \\
2.22\end{array}$ & $\begin{array}{l}\text { Seated / } \\
\text { Standing / } \\
\text { Wheelchair }\end{array}$ & $\begin{array}{l}5 \times 5 ; 10 \times \\
5 ; 10 \times 8 ; 15 \\
\times 8 ; 15 \times 13\end{array}$ & N/A & No & \\
\hline PreXion & Explorer & $\begin{array}{l}\text { CsI / } \\
\text { aSi }\end{array}$ & $90-110$ & $1-5$ & $\begin{array}{l}2.7 \\
\mathrm{mmAl}\end{array}$ & 0.3 & $0.074-0.300$ & $10-20$ & $5-10$ & Pulsed & $<60$ & 360 & 16 & $\begin{array}{l}1.56 \\
\times \\
1.11 \\
\times \\
2.27\end{array}$ & $\begin{array}{l}\text { Seated / } \\
\text { Standing / } \\
\text { Wheelchair }\end{array}$ & $\begin{array}{l}5 \times 5 ; 10 \times \\
10 ; 15 \times 8 \\
15 \times 16\end{array}$ & 185 & No & \\
\hline QR Systems & NewTom VGi evo & $\begin{array}{l}\text { CsI / } \\
\text { aSi }\end{array}$ & 110 & $1-32$ & $\begin{array}{l}12 \\
\text { mmAl }\end{array}$ & $\begin{array}{l}0.3 \\
/ \\
0.6\end{array}$ & $0.100-0.300$ & $15-25$ & $0.9-6$ & Pulsed & $<60$ & 360 & 16 & $\begin{array}{l}1.64 \\
\times \\
1.29 \\
\times \\
2.33\end{array}$ & $\begin{array}{l}\text { Seated / } \\
\text { Standing / } \\
\text { Wheelchair }\end{array}$ & $\begin{array}{l}5 \times 5 ; 8 \times 5 \\
8 \times 8 ; 10 \times \\
5 ; 10 \times 10 \\
12 \times 8 ; 15 \times \\
5 ; 15 \times 12 \\
16 \times 16 ; 24 \\
\times 19\end{array}$ & 377 & No & $\begin{array}{l}\text { Scout image, } \\
\text { mA-modulation, } \\
\text { MAR }\end{array}$ \\
\hline QR Systems & NewTom 5G XL & $\begin{array}{l}\text { CsI / } \\
\mathrm{aSi}\end{array}$ & 110 & $1-32$ & $\begin{array}{l}11.2 \\
\mathrm{mmAl}\end{array}$ & 0.3 & $0.075-0.300$ & $18-36$ & $0.9-9$ & Pulsed & $<60$ & 360 & 16 & $\begin{array}{l}3.61 \\
\times \\
1.75 \\
\times \\
1.80\end{array}$ & $\begin{array}{l}\text { Supine / } \\
\text { seated } \\
\text { (orthopaedic) }\end{array}$ & $\begin{array}{l}6 \times 6 ; 8 \times 5 \\
8 \times 8 ; 10 \times \\
5 ; 10 \times 10 \\
12 \times 8 ; 15 \times \\
5 ; 15 \times 12 ; \\
15 \times 22 ; 18 \\
\times 16 ; 21 \times \\
19\end{array}$ & 660 & No & $\begin{array}{l}\text { Scout image, } \\
\text { mA-modulation, } \\
\text { MAR }\end{array}$ \\
\hline QR Systems & NewTom 7G Body & $\begin{array}{l}\text { CsI / } \\
\mathrm{aSi}\end{array}$ & $70-120$ & $5-120$ & $\begin{array}{l}21 \\
\mathrm{mmAl}\end{array}$ & $\begin{array}{l}0.3 \\
/ \\
0.6\end{array}$ & $0.090-0.500$ & $7.2-26$ & $1.4-8.8$ & Pulsed & $<60$ & 360 & 16 & $\begin{array}{l}3.94 \\
\times \\
2.05 \\
\times \\
2.08\end{array}$ & $\begin{array}{l}\text { Supine / } \\
\text { seated } \\
\text { (orthopaedic) }\end{array}$ & $\begin{array}{l}4 \times 4 ; 6 \times 6 \\
8 \times 6 ; 8 \times 8 \\
10 \times 10 ; 13 \\
\times 6 ; 13 \times 8 \\
13 \times 12 ; 15 \\
\times 6 ; 13 \times \\
17 ; 13 \times 32 \\
17 \times 12 ; 17\end{array}$ & 1050 & No & $\begin{array}{l}\text { Scout image, } \\
\text { mA-modulation, } \\
\text { MAR }\end{array}$ \\
\hline
\end{tabular}




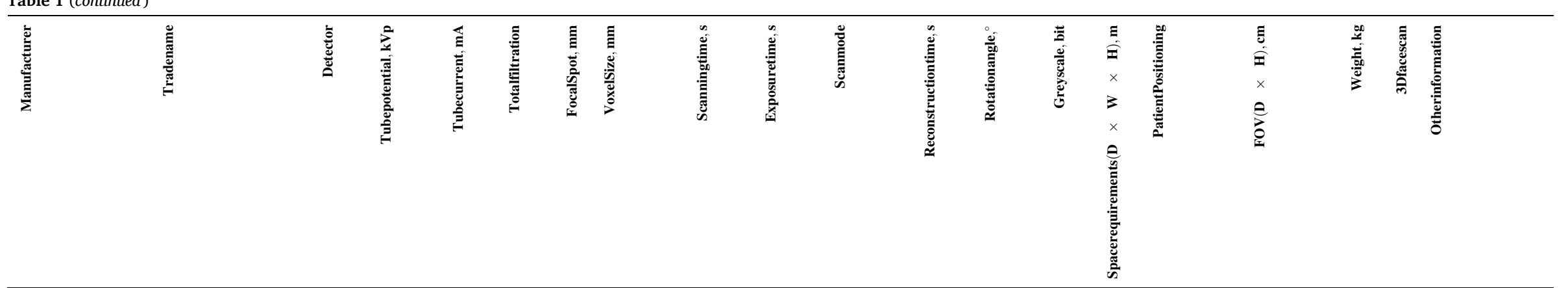

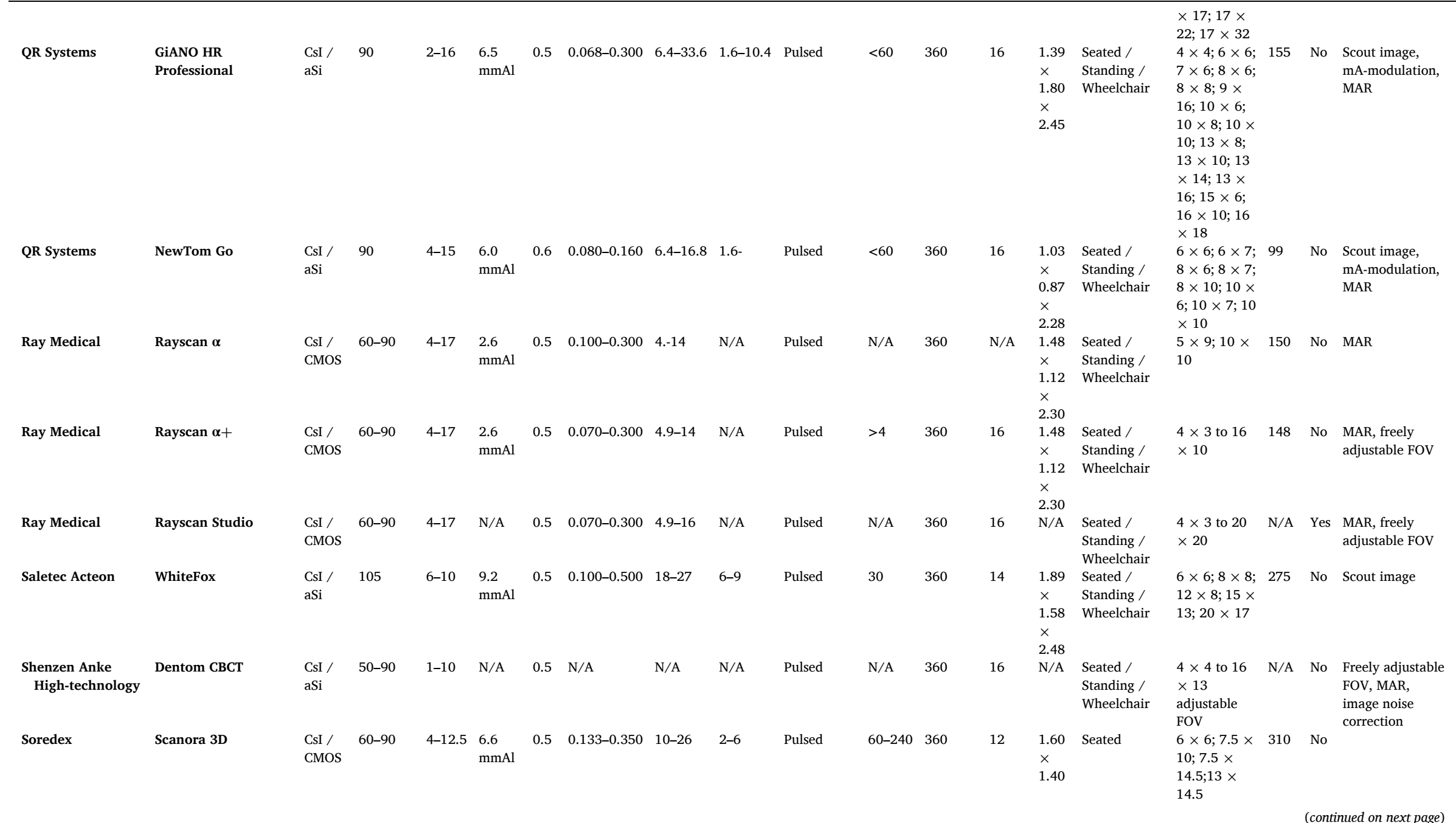




\begin{tabular}{|c|c|c|c|c|c|c|c|c|c|c|c|c|c|c|c|c|c|c|c|}
\hline 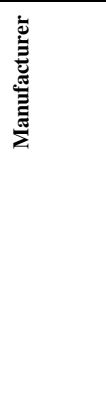 & 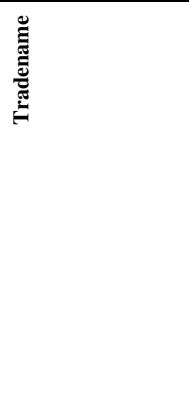 & 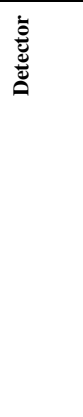 & 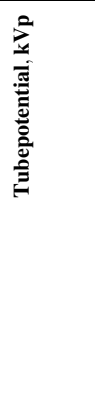 & 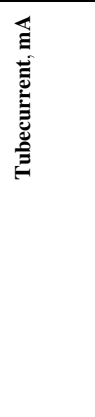 & 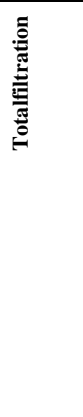 & 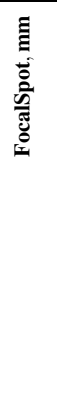 & 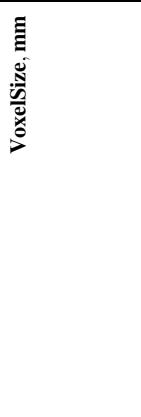 & 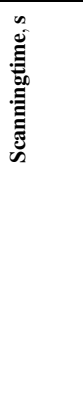 & 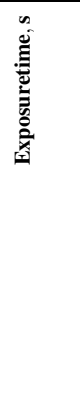 & 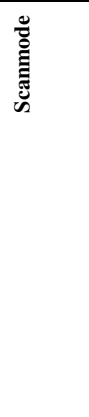 & 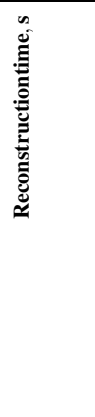 & 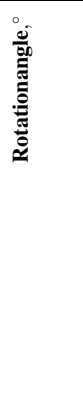 & 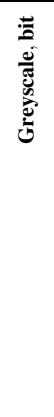 & 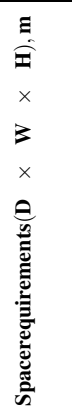 & 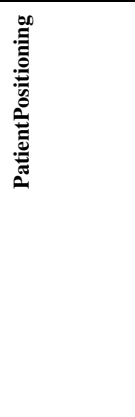 & 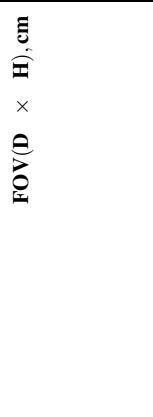 & 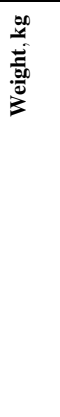 & 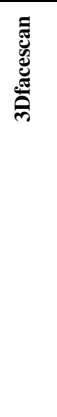 & 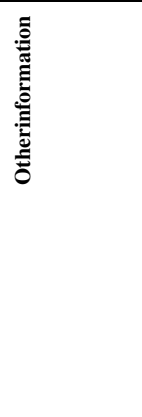 \\
\hline Soredex & Scanora 3Dx & $\begin{array}{l}\text { CsI / } \\
\text { aSi }\end{array}$ & $60-90$ & $4-10$ & $\begin{array}{l}6.6 \\
\text { mmAl }\end{array}$ & 0.5 & $0.100-0.500$ & $18-34$ & $2.4-6$ & Pulsed & $60-240$ & 360 & 14 & $\begin{array}{l}\times \\
\times \\
1.97 \\
1.60 \\
\times \\
1.40 \\
\times \\
1.97\end{array}$ & Seated & $\begin{array}{l}5 \times 5 ; 5 \times \\
10 ; 8 \times 10 ; 8 \\
\times 16.5 ; 14 \\
\times 10 ; 14 \times \\
16.5 ; 18 \times \\
16.5 ; 24 \times \\
16.5\end{array}$ & 310 & No & \\
\hline Soredex & Cranex 3D & $\begin{array}{l}\text { CsI / } \\
\text { CMOS }\end{array}$ & $60-90$ & $4-12.5$ & $\begin{array}{l}3.2 \\
\text { mmAl }\end{array}$ & 0.5 & $0.133-0.350$ & $10-26$ & $2.3-6$ & Pulsed & $60-240$ & $\begin{array}{l}180 / \\
360\end{array}$ & 14 & $\begin{array}{l}1.60 \\
\times \\
1.40 \\
\times \\
1.97\end{array}$ & $\begin{array}{l}\text { Standing / } \\
\text { Seated / } \\
\text { Wheelchair }\end{array}$ & $\begin{array}{l}6 \times 6 ; 7.5 \times \\
10 ; 7.5 \times \\
14.5 ; 13 \times \\
14.5\end{array}$ & 200 & No & \\
\hline Soredex & Cranex 3Dx & $\begin{array}{l}\text { CsI / } \\
\text { CMOS }\end{array}$ & $57-90$ & $4-16$ & $\begin{array}{l}3.2 \\
\text { mmAl }\end{array}$ & 0.5 & $0.085-0.400$ & $10-40$ & $1-9$ & Pulsed & $60-240$ & $\begin{array}{l}180 / \\
360\end{array}$ & 14 & $\begin{array}{l}1.96 \\
\times \\
1.41 \\
\times \\
2.41\end{array}$ & $\begin{array}{l}\text { Standing / } \\
\text { Seated / } \\
\text { Wheelchair }\end{array}$ & $\begin{array}{l}5 \times 5 ; 6.1 \times \\
7.8 ; 7.8 \times \\
7.8 ; 7.8 \times \\
15 ; 13 \times 15\end{array}$ & 200 & No & \\
\hline Trident & X-View 3D PAN & $\begin{array}{l}\text { CsI / } \\
\text { CMOS }\end{array}$ & $61-85$ & $4-10$ & $\begin{array}{l}2.5 \\
\text { mmAl }\end{array}$ & 0.5 & 0.160 & 15 & 9.2 & $\mathrm{~N} / \mathrm{A}$ & $>10$ & 230 & 14 & $\begin{array}{l}1.07 \\
\times \\
1.72 \\
\times \\
2.23\end{array}$ & $\begin{array}{l}\text { Standing / } \\
\text { Seated / } \\
\text { Wheelchair }\end{array}$ & $8.5 \times 8.5$ & 125 & No & \\
\hline Vatech & Green $16 / 18$ & $\begin{array}{l}\text { CsI / } \\
\text { CMOS }\end{array}$ & $60-99$ & $4-16$ & N/A & 0.5 & $0.080-0.300$ & $4.9-9$ & N/A & N/A & $\mathrm{N} / \mathrm{A}$ & 360 & 14 & $\begin{array}{l}1.49 \\
\times \\
1.13 \\
\times \\
2.17\end{array}$ & $\begin{array}{l}\text { Seated / } \\
\text { Standing / } \\
\text { Wheelchair }\end{array}$ & $\begin{array}{l}5 \times 5 ; 8 \times 9 \\
12 \times 9 ; 13 \times \\
10 ; 16 \times 9 \\
18 \times 10\end{array}$ & 134 & No & MAR \\
\hline Vatech & Green 21 & $\begin{array}{l}\text { CsI / } \\
\text { CMOS }\end{array}$ & $60-120$ & $4-10$ & $\mathrm{~N} / \mathrm{A}$ & 0.5 & $0.200-0.400$ & 18 & $\mathrm{~N} / \mathrm{A}$ & $\mathrm{N} / \mathrm{A}$ & $\mathrm{N} / \mathrm{A}$ & 360 & 14 & $\begin{array}{l}1.57 \\
\times \\
1.53 \\
\times \\
2.18\end{array}$ & $\begin{array}{l}\text { Seated / } \\
\text { Wheelchair }\end{array}$ & $\begin{array}{l}8 \times 8 ; 12 \times \\
9 ; 17 \times 11 \\
17 \times 15 ; 21 \\
\times 19\end{array}$ & 321 & Yes & \\
\hline Vatech & Pax-i3D & $\begin{array}{l}\text { CsI / } \\
\text { CMOS }\end{array}$ & $50-90$ & $4-10$ & $\mathrm{~N} / \mathrm{A}$ & 0.5 & $0.120-0.300$ & $15-24$ & $\mathrm{~N} / \mathrm{A}$ & $\mathrm{N} / \mathrm{A}$ & $\mathrm{N} / \mathrm{A}$ & 360 & 14 & $\begin{array}{l}1.29 \\
\times \\
1.12 \\
\times \\
2.34\end{array}$ & $\begin{array}{l}\text { Seated / } \\
\text { Standing / } \\
\text { Wheelchair }\end{array}$ & $\begin{array}{l}5 \times 5 ; 8 \times 5 \\
8 \times 8 ; 12 \times 9\end{array}$ & $\mathrm{~N} / \mathrm{A}$ & No & \\
\hline Vatech & Pax-i3D Green & $\begin{array}{l}\text { CsI / } \\
\text { CMOS }\end{array}$ & $50-100$ & $4-16$ & $\begin{array}{l}2.8 \\
\text { mmAl }\end{array}$ & 0.5 & $0.080-0.300$ & $5.9-15$ & $\mathrm{~N} / \mathrm{A}$ & Pulsed & $13-212$ & 360 & 14 & $\begin{array}{l}1.33 \\
\times \\
1.20 \\
\times \\
2.34\end{array}$ & $\begin{array}{l}\text { Seated / } \\
\text { Standing / } \\
\text { Wheelchair }\end{array}$ & $\begin{array}{l}5 \times 5 ; 8 \times 5 \\
8 \times 8 ; 12 \times \\
9 ; 17 \times 15\end{array}$ & 182 & No & $\begin{array}{l}\text { Scout image, } \\
\text { MAR }\end{array}$ \\
\hline
\end{tabular}




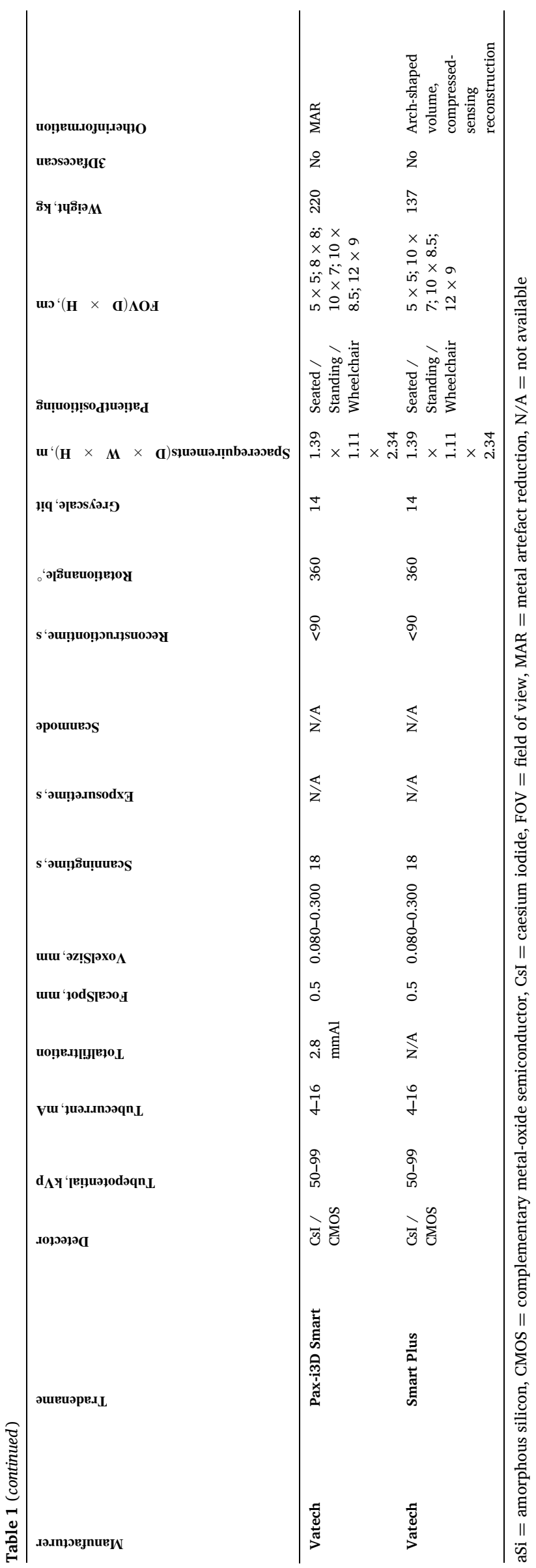

resolution and delineation of smaller fine structures, such as in dental trauma cases. Not only voxel size but also the focal spot size affects spatial resolution. Most of the recent CBCT systems utilise a focal spot size of $0.5 \mathrm{~mm}$ (range 0.2 to $0.7 \mathrm{~mm}$ ). The smaller the focal spot size, the smaller the penumbra at the detector, which results in a sharper image [25].

\subsection{Exposure settings}

Most of the dental CBCT devices use a pulsed x-ray beam exposure, although a few vendors still utilise a continuous radiation exposure. Usually, the pulsed irradiation results in lower patient doses than continuous exposure mode, as the radiation is turned off intermittently during the image acquisition process. Only a few CBCT systems use fixed tube current and tube potential for 3D imaging, whereas most scanners enable these to be changed in certain limits, resulting in better possibilities for scan optimisation in terms of radiation dose and image quality. Typically, tube current values selectable for 3D imaging are between 2 and $15 \mathrm{~mA}$, but the range covering all units is 1 to $120 \mathrm{~mA}$. Tube voltages range from 50 to $120 \mathrm{kVp}$, with typical values between 60 and $90 \mathrm{kVp}$. However, the lower $\mathrm{kVp}$ values may not always be available in CBCT scans, but only in the panoramic or cephalographic imaging modes. As in multi-slice CT scanners, the $\mathrm{kV}$ optimisation would also be beneficial in СВCT scanning. The total $x$-ray tube filtration of the equipment that affects the $\mathrm{x}$-ray spectra varies remarkably (from 2.5 mm-Al at minimum up to $21 \mathrm{~mm}-\mathrm{Al}$ [NewTom 7G Body, QR Systems srl, Verona, Italy]). Additionally, more CBCT vendors are currently offering an additional copper filtration in their newest systems, whereas 6 years ago this was only available in the Planmeca CBCT systems. The additional copper filtration is used to harden the photon beam and to reduce patient dose. The set of $\mathrm{x}$-ray spectra from the scanners is presented in Fig. 2.

\subsection{Scan time}

The scanning times of current СBCT vary between 2 and $45 \mathrm{~s}$, depending, for example, on the acquired number of projections and rotation arc of the scan. The exposure times are typically less than this because of the pulsed irradiation and ranges from 0.5 to $40 \mathrm{~s}$.

\subsection{Image reconstruction}

The reconstruction technique still mostly used in dental CBCT systems utilises 3D filtered back projection (FBP) by Feldkamp-Davis-Kress (FDK) algorithm [25] due to its simplicity and rapid reconstruction times. However, as discussed previously [5], СBCT, with its limited amount of projections, shorter angular range in data acquisition, and truncated data could benefit considerably from more advanced iterative reconstruction techniques. Recently, some CBCT models have been introduced that use iterative reconstruction methods (X1 Scancomfort, 3Shape A/S, Copenhagen, Denmark) or DL-based reconstruction methods (Smart3D-X scanner, LargeV Instrument Corp Ltd., Beijing, China). The reconstruction times differ between the scanners from almost real-time reconstructions up to a few minutes, depending on the acquisition parameters (FOV, voxel size, number of projections, rotation angle), hardware (processing speed, data transfer from acquisition to reconstruction computer), and software (reconstruction algorithms) used.

\subsection{Metal-artefact reduction techniques}

Metal restorative materials (e.g. dental implants, metal fillings and crowns, fixed orthodontic appliances) and related artefacts are common in dental imaging. Metal implants result in streaking and beamhardening artefacts in the CBCT images that can be seen as dark shading and bright streaks, especially around highly attenuating 
metallic objects within the image volume [37]. However, many dental CBCT systems currently have noise/scatter correction and metal-artefact reduction algorithms to overcome the decreased image quality due to highly attenuating metal implants.

\section{Quality assurance}

Optimisation of radiological imaging equipment should span the wider context of quality management and quality assurance (QA) to connect the optimal setting of examination parameters to technical conformance of the scanners. This also applies to dental CBCT. QA process and quality control (QC) tests form important part of medical imaging quality and safety in any radiological imaging method. QA aims to assure that the technical performance and vendor specifications of the equipment are continuously maintained in clinical use and through the equipment life cycle. QC is a key part of QA to periodically assess that quality requirements are met. On a more general level, QA and QC also aim to reduce uncertainties and errors and to reduce the likelihood of accidents and incidents.

Manufacturers have specific QA programmes for imaging equipment and should conform to applicable standards, such as those provided by DIN (German institute for standards) [38,39] and guidelines such as published by SEDENTEXCT [40] and European Federation of Organisations for Medical Physics (EFOMP) [41]. However, clinical staff should also be aware of the testing needs and perform regular QA tests, as required for the user and to monitor the constancy of their equipment. Thus, the QA and QC activities and responsibilities cover vendor, service, and users as connected shareholders in the overall quality and safety of imaging. While focusing on the clinical use of CBCT scanners, image quality evaluation is a particularly important part of the overall QA programme and should be integrated together with radiation exposure and technical performance monitoring for all CBCT scanners regardless of specific application area.

Without going into details of QA/QC testing procedures, typical image quality tests focus on the following properties: image grayscale values (CT numbers), image uniformity, (high contrast) spatial resolution, low-contrast resolution (contrast-to-noise ratio [CNR]), image noise, artefacts, and geometric precision. Scanner calibrations related to image quality testing typically include geometric and detector pixel calibration. Testing of radiation output typically includes measurements of KAP or DAP and air kerma at the focus-to-detector distance and in phantom dose indicators (e.g. traditional CTDI). It should be emphasised that possible Hounsfield Unit (HU) scaling in dental CBCT is only indicative and cannot be accurately calibrated as compared to multislice CT.

As an example of the practical dental and ear, nose, and throat (ENT) radiology CBCT (Instrumentarium OP300) user level image QA, phantom testing is performed quarterly based on vendor phantoms and integrated QA and calibration application (the vendor advises a 6-month frequency for the phantom scan procedure). Image quality tests mainly follow the previously mentioned typical targets. Three separate phantoms are used for image quality tests, including 3D calibration phantom, QC phantom, and constancy test phantom (Fig. 3).

The resulting QA/QC documentation may be provided by automatic log files and images. Spatial resolution can be determined subjectively from line-pair pattern or objectively from a point source providing highcontrast impulse response function in the form of an MTF curve (as a function of spatial frequencies).

Technical QA procedures of dental CBCT may offer automatic features for QA monitoring and analysis purposes. By an integrated and automatic QA software, the regular CBCT QA process can be streamlined and does not require detailed technical knowledge from the users. However, in addition to the scanner image QA phantom testing, the users also perform regular display monitor QA, which ensures that the overall image review process chain is covered in the QA programme and conforms to the general radiology QA programme (of all modalities) for consistency. In addition to this user-level regular testing, further technical-level testing of the scanner is performed during regular maintenance service visits by the vendor, including $\mathrm{x}$-ray tube and radiation output specific parameters.

\section{Patient dose and optimisation aspects}

The overarching principles of optimisation and justification have been present in the radiation protection system since its establishment in the ICRP publication 26 [42], from where they have later been adopted to international and European safety standards $[43,44]$. In medical use of radiation, justification requires that the radiological procedure must improve the diagnosis and treatment of patients. Moreover, an individual patient must benefit from that particular exposure, considering the specific characteristics of the exposed person. Therefore, justification requires weighing the benefits and possible harm caused by the exposure. In medical exposures, the harmful effects of ionizing radiation are minimised as far as practically possible to maximise the net benefit to the patient (i.e. the patient exposures are optimised).

Optimisation starts from a proper selection of equipment, competent and trained operators, and day-to-day working methods. QA processes must also be established to ensure compliance with regulations and proper functioning of the equipment. Optimisation of the exposure should be done at the level of individual patient, such as through selection of the appropriate imaging modality, x-ray tube voltage, filtration, exposure time, tube current, field size, and voxel size and from information from earlier examinations. In recent CBCT devices, lowdose protocols have become increasingly common (see a recent review for a discussion of possibilities and limitations [45]). However, the lack of automatic exposure control in dental CBCT devices has limited optimisation efforts. This has also been noted in the ICRP publication 129 [46] and discussed in relation to the recent EURADOS survey [47]. Thus, on-site optimisation of imaging parameters is of great importance. In the optimisation process, a multi-professional team is a prerequisite for optimal results, as technical, diagnostic, and dosimetric aspects must be considered (see e.g. ICRP Task Group 108, https://www.icrp.org).

Differences in radiation dose levels are large not only between various CBCT devices from different vendors but also within the same scanner models $[14,35,48,49]$. The same authors also stated that a 50 fold difference in radiation exposure to a patient can easily be observed when changing the settings in specific CBCT equipment. Widely varying non-optimised doses were also found for example in the SEDENTEXCT research project [40] and in the European DIMITRA (dentomaxillofacial paediatric imaging: an investigation toward lowdose radiation induced risks) project [50]. A recent study by the IAEA [51] revealed that optimisation in dental and maxillofacial CBCT imaging was still not at a mature stage, 7 years after the publication of SEDENTEXCT guidelines. This conclusion was also supported by a survey performed by the EURADOS working group 12 [47].

\section{Dosimetry and use of diagnostic reference levels}

CBCT dosimetry still lacks a consensus on dose metrics at a practical level (KAP vs. CTDI, or some other dose quantity) [47,51]. This may also have implications on optimisation, as comparisons of dose levels between clinics, or even between different vendors or models, may be difficult. We have discussed CBCT dosimetry issues and related challenges in our previous publication [5]. To fulfil the need for practical guidance in QC of CBCT devices, the joint EFOMP-ESTRO-IAEA protocol was published in 2017 [52]. The protocol is applicable to all CBCT devices and is described at a practical level that can be directly applied in the clinics. The topics cover the assessment of image quality and radiation output of the device and include a discussion on image quality phantoms.

Several studies have investigated radiation exposures to patients from dentomaxillofacial CBCT examinations. Effective and organ doses 
have been determined using thermoluminescent dosimeters (TLD), optically stimulated luminescence (OSL) dosimeters, and metal-oxidesemiconductor field-effect transistor (MOSFET) detectors [53-55]. Recent studies have also used Monte Carlo simulations to estimate effective and organ doses [53,55-57]. The magnitudes of these biological dose quantities vary remarkably in dental CBCT depending on the FOV size, positioning, and exposure parameters. An example of a 3D dose map produced with a Monte Carlo simulation is shown in Fig. 4.

\subsection{Conversion coefficients from DAP to effective dose}

Effective doses can also be estimated using appropriate dose conversion factors from DAP or KAP. Mah et al. reported in their review article the usage and magnitudes of effective dose conversion coefficients for dental CBCT equipment and examinations [49]. They reported that conversion coefficients range from 0.035 to $0.31 \mu \mathrm{Sv}$ / $\mathrm{mGycm}^{2}$ with a mean value of $0.129 \mu \mathrm{Sv} / \mathrm{mGycm}^{2}$. They found FOV size significantly affects the obtained conversion coefficients and depends in part on the relative contribution of thyroid and salivary glands and their inclusion in the primary beam.

For the current review, we also performed Monte Carlo simulations to provide conversion coefficients from DAP $\left(\mathrm{mGycm}^{2}\right)$ to effective dose $(\mu \mathrm{Sv})$ with various scan scenarios. Our simulations were motivated by the fact that highly variable FOV size, spectral characteristics, and scan modes involving applied mAs (by pulsed exposure and $\mathrm{mA}$ levels) and angular scan range (typically between $180^{\circ}$ and $360^{\circ}$ in axial plane) in different scanner models make it difficult to report effective doses comprehensively and also specifically for relevant dental CBCT indications. The variable scan scenarios in our Monte Carlo simulations covered three different FOV sizes, three x-ray photon spectra, variable anatomical FOV positions (mainly focusing on small FOV), and angular scan range (Table 2). Three x-ray spectral qualities were chosen to represent soft, medium, and hard x-ray beam spectrum produced by low, medium, and high added filtrations, respectively (adapted from the data in Fig. 2), corresponding to different scanner models. Three FOV sizes were chosen to represent typical small, medium, and large FOVs (Table 1). Anatomical FOV positions were selected by the relevant main indications for the scan of third molar (wisdom tooth) on the lateral side, upper and lower canine in the antero-lateral position, and anterior centre position of the jaw. Medium FOV was positioned in the lateral and anterior position due to larger coverage of the dental structures in mandible and maxilla in the same scan. Large FOV was centred for whole skull indication. All simulations were performed with $360^{\circ}$ rotation with additional simulations using $200^{\circ}$ latero-posterior rotation for selected FOV and anatomical positions (third molar small FOV and whole skull large FOV). Simulations were performed with PCXMC 2.0 rotational version (STUK, Helsinki, Finland). As a necessary part of the effective dose conversion coefficient calculations, organ doses were also determined and thyroid dose contribution was given a specific emphasis due to the relevance for shielding aspects.

As shown in Table 2, the DAP to effective dose conversion coefficients vary from 0.06 to $0.3 \mu \mathrm{Sv} / \mathrm{mGycm}^{2}$ with an average of 0.18 $\mu \mathrm{Sv} / \mathrm{mGycm}^{2}$. Thus, our conversion coefficients from DAP to effective dose are also consistent with those reported by Mah et al. [49]. A wholeskull scan with large FOV $(20 \times 20 \mathrm{~cm})$ applying soft spectrum and partial rotation represented the lowest conversion coefficients. The highest conversion coefficients were found with the highest photon energy spectrum and small FOV, demonstrating the contribution of scatter with higher photon energies and dose collection from partially exposed tissue outside of the FOV. It should also be noted that the wholeskull scan does not have partially exposed tissue outside of the axial scan FOV, although the scatter from its larger FOV extends further in the vertical (craniocaudal) anatomical direction. The relative contribution of thyroid dose to effective dose varied from $3 \%$ to $16 \%$ with an average of $8 \%$. Contributions around $10 \%$ were related to whole-skull exposure with higher photon energy spectrum, and the highest contributions extending to $16 \%$ were related to canine scans of the mandible (lower jaw), where partial exposure had most coverage to the thyroid position. Upper estimates of the thyroid effective dose relative contribution were additionally determined from two medium-spectrum simulations with $200^{\circ}$ and $360^{\circ}$ rotation, which extended the scan FOV $(26 \mathrm{~cm}$ diameter $\times 35 \mathrm{~cm}$ height) to include the thyroid entirely within the direct exposure (against the appropriate imaging practice). These simulations revealed a $35 \%$ contribution of thyroid dose to effective dose in $200^{\circ}$ posterior rotation and $43 \%$ contribution in $360^{\circ}$ full rotation, respectively. These results emphasise the general recommendation to limit the scan FOV by size and anatomical positioning to avoid direct thyroid exposure.
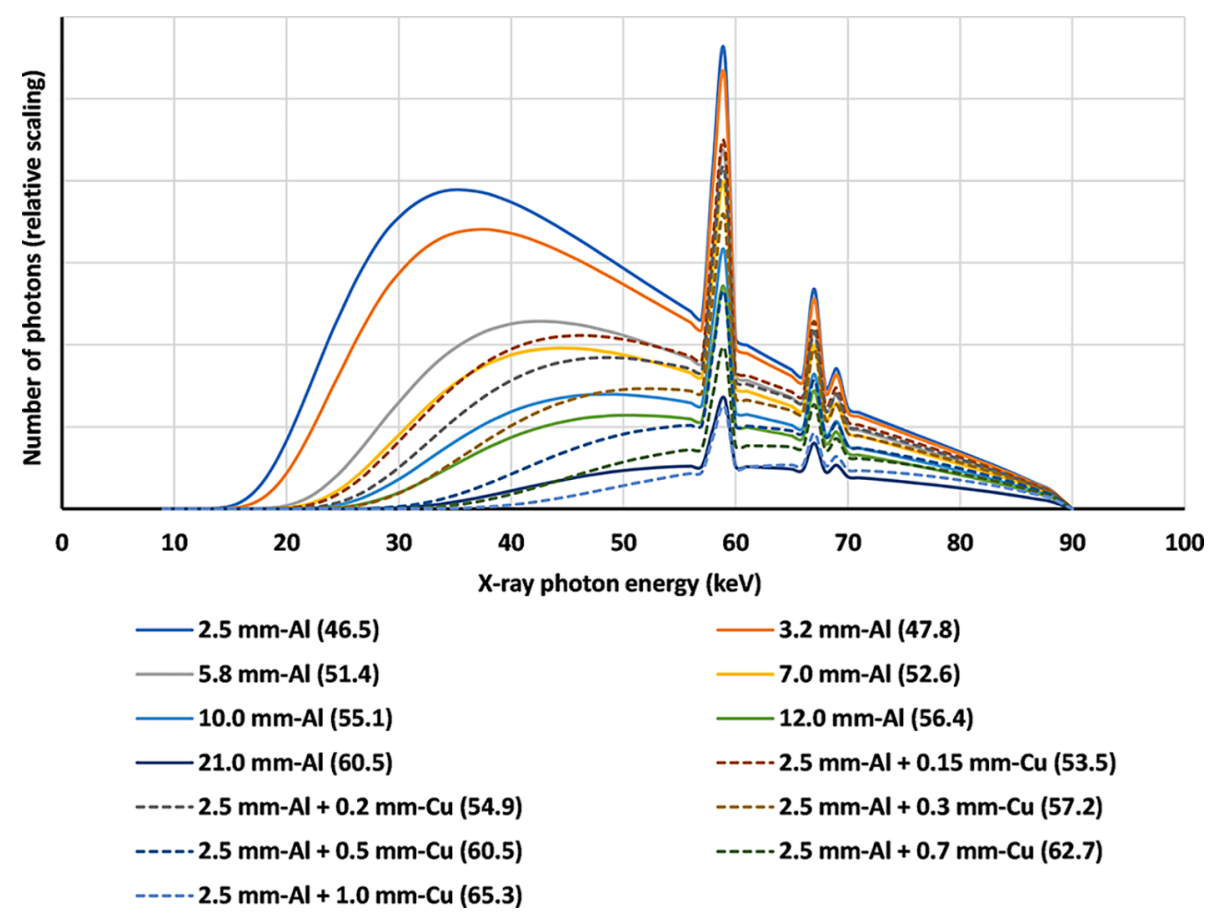

Fig. 2. X-ray spectra from CBCT scanners referenced with $90-\mathrm{kVp}$ tube voltage and typical $10^{\circ}$ anode angle taking into account the total applied filtration by aluminium (solid lines) or combined aluminium and copper (dash lines). Mean photon energy (keV) of each spectrum is marked in parentheses on the spectrum labels. As anticipated, increase in total filtration will significantly affect the spectral shape and mean energy of the beam. Furthermore, added copper filtration clearly reduces the lower energy part of the spectrum. 


\subsection{Diagnostic reference levels}

Diagnostic reference levels (DRLs) have been introduced as a practical tool to facilitate optimisation. While the DRLs do not classify patient exposures as good or bad, they contribute toward good imaging practices by indicating exceptionally high (or low) exposures. The European Basic Safety Standard [44] requires that "Member States shall ensure the establishment, regular review and use of diagnostic reference levels for radiodiagnostic examinations". However, very few countries currently have established national DRLs in dental CBCT imaging (Table 3, see also [58] for a general discussion on optimisation and DRLs). Switzerland published their national DRLs for five dental indications [59] as did Finland for four dental or maxillofacial indications [60] and Sweden for three non-indication-based examinations in the dental region [61]. In the UK, Public Health England has suggested DRLs both for a specific adult and child CBCT protocol [62]. The DRL can be established at several levels, from European (regional) to national and local (clinic). However, as dental CBCT equipment are often in small private clinics that may not have access (although required by legislation [44]) to medical physics expert service, national and regional efforts are needed to ensure widespread application of DRLs.

\section{Clinical aspects}

In $2 \mathrm{D}$ radiographs, the superimposition of different anatomical structures and geometric distortion may impair image interpretation. This can be avoided using 3D imaging (Figs. 5 and 6). If a 3D examination provides new information compared to conventional 2D imaging and impacts treatment decisions or planning, it can be considered justified. Despite recent advances in CBCT, radiation doses of low-dose imaging protocols are still often higher than in conventional intraoral, panoramic, or cephalometric radiographs. Therefore, CBCT imaging indications and referral criteria are of utmost importance in clinical practice $[46,63,64]$.

\subsection{Guidelines}

Since the introduction of the CBCT technique in dental and maxillofacial imaging in the late $1990 \mathrm{~s}$, the practitioners saw a long period of non-existent protocols or guidelines on how to approach optimisation and patient protection in clinical practice. Guidelines were first established at the international level by the American Academy of Oral and Maxillofacial Radiology in 2008 [65] and the European Academy of Dental and Maxillofacial Radiology (EADMFR) in 2009 [66], and further complemented by the EU-funded SEDENTEXCT consortium with its Safety and Efficacy of a New and Emerging Dental X-ray Modality research project in 2012 [40]. In addition to the European guidelines, national guidelines from different countries are also available, including a recent review compiling and summarising the CBCT-related guidelines from organisations and associations within North America [8,40,67]. Although the SEDENTEXCT panel members stated that especially the referral criteria should be reviewed and updated at intervals of no $>5$ years, financial support for reviewing the guidelines is unfortunately still insufficient $[40,68]$. However, during the past 8 years, several systematic reviews and position statements focusing on various maxillofacial imaging indications of СBCT have been published.

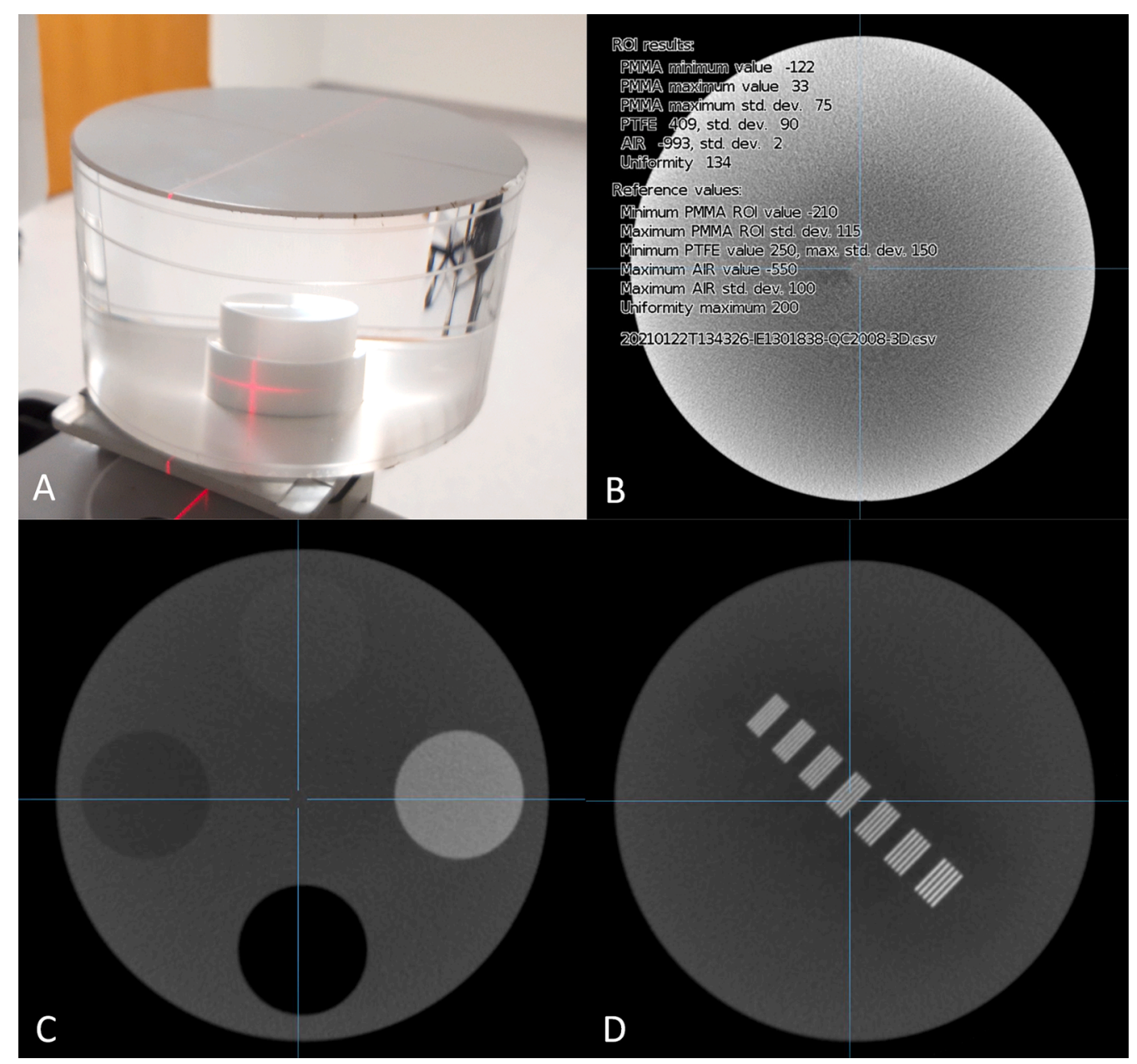

Fig. 3. Example of QC and constancy phantom scans and resulting axial images from Instrumentarium OP300 CBCT scanner: a) a constancy phantom positioned for the scan, b) an axial image of the uniform section of the constancy phantom with automatic reporting of the acceptance of the test results covering the material-specific contrast and noise values in addition to uniformity value, c) an axial image showing the different material cylinders in the QC phantom and d) an axial image of the high contrast line-pair patterns for the visual evaluation of the spatial resolution. Approximation of CT numbers (in HU; Hounsfield units) in density calibration is demonstrated with a fairly wide tolerance of materialspecific densities, e.g. -200 to +200 HU for acryl (PMMA) voxel values. It should be noted that this range of allowed density variation is over an order of magnitude greater than in typical multi-slice CT scanners. 


\subsection{CBCT imaging indications}

As children and young people are at greater risk of adverse effects of ionizing radiation, special care must be taken when imaging these patient groups [69]. However, there are some indications that the optimisation is insufficiently performed in the case of young patients [50]. Therefore, the European DIMITRA project proposed an ALADAIP principle (As Low as Diagnostically Acceptable being Indication-oriented and Patient-specific) when imaging children [50]. In paediatric dentistry and orthodontics, impacted or supernumerary teeth frequently need surgical intervention. CBCT scanning is justified if the exact position of the tooth, its relationship with intimate anatomical structures, and possible resorption of neighbouring teeth cannot reliably be assessed from $2 \mathrm{D}$ radiographs or if CBCT imaging will otherwise influence treatment planning $[50,70]$. According to the updated EADMFR recommendation, preoperative CBCT imaging should not be used routinely to evaluate the relationship with mandibular third molars (wisdom teeth) and the mandibular canal, but only if the surgeon has a specific clinical question that cannot be answered by panoramic or intraoral imaging [68]. Traumatic or congenital loss of permanent teeth can be treated with an autotransplantation operation and the dimensions of both the tooth transplant and the recipient site bone volume can be measured reliably from CBCT images. If orofacial cleft (lip and/or palate cleft) operations need volumetric assessment of bone defect, CBCT scanning can replace medical multi-slice CT scanning before a bone-grafting operation. Craniofacial syndromes, skeletal asymmetries requiring surgical intervention, and orthognathic surgery are other possible indications for CBCT imaging. One significant clinical disadvantage of CBCT imaging is the longer scanning time compared to conventional imaging. Therefore, cooperation of paediatric patients must be considered. Movement of the patient during the scanning time can produce image artefacts and impair image quality $[50,69,71,72]$.

The root canal anatomy of a single tooth may be complex, especially in multirooted molars and premolars, and all 2D radiographs have limitations in depicting morphological details of teeth and the surrounding alveolar bone structure. The European Society of Endodontology has published position statements in 2014 [73] and 2019 [74] on the use of CBCT in endodontics. It concluded that CBCT imaging should be considered in the following cases: suspicion of periapical pathoses when $2 \mathrm{D}$ radiographs are inconclusive and the patient has contradictory symptoms, assessment of root canal anatomy prior to initial endodontic treatment and in re-treatment cases, and prior to periradicular surgery [74]. Dental trauma, especially horizontal root fractures, and possible later complications such as resorptions and root canal obliterations, and developmental tooth anomalies can be accurately diagnosed in CBCT images $[69,74]$. In endodontic and dental trauma imaging, a higher spatial resolution may be required to delineate complex and detailed structures, such as root canals and fractures. However, it is good to remember that smaller voxels capture fewer x-ray photons and therefore provides more noise to the images, which may lead to use of increased radiation dose levels in scanning. We remind readers that the voxel size should be selected according to clinical indication. Additionally, although the theoretical spatial resolution of CBCT devices may be high due to small focal spot and voxel sizes, beam projection geometry, scatter, patient movement, detector motion blur and fill factor, number of projections, and reconstruction algorithms also impact the final spatial resolution and thus the capabilities of viewing detailed structures. As CBCT scanning can last from a few seconds up to $45 \mathrm{~s}$, the imaging is vulnerable to patient motion. As discussed previously, the heart beating alone can induce 80-90 $\mu \mathrm{m}$ movement of the patient's head $[31,33]$.

In a recent nationwide survey conducted in co-operation with the Finnish Radiation and Nuclear Safety Authority, the most common imaging indication was preoperative implant planning in the facilities performing CBCT examinations of the head and neck region in Finland [75]. Before a surgical operation, alveolar bone volume, restricting anatomical structures (e.g. sinuses, nerve canals), bone structure, and alveolar ridge morphology must to be assessed. Cross-sectional imaging is needed if clinical examination and conventional imaging are insufficient. CBCT imaging is especially advisable before bone augmentation and sinus floor elevation operations. Successful occlusal rehabilitation with implants involves both surgical and prosthetic planning. In computer-guided planning, both CBCT data and digital impression are transferred to a software and the position of the implant in relation to occlusion can be planned virtually. After that, surgery can be performed with custom-made stereolithographic guides. Post-operative imaging with CBCT is restricted to specific complications, such as unexplained pain and infections. Metal restorations and implants can cause image artefacts and have a negative impact on image quality [76-78].

Only osseous structures can reliably be assessed when imaging the temporomandibular joint (TMJ). Soft tissues and intra-articular anatomy should be evaluated from magnetic resonance images. The diagnostic benefit of CBCT images and impact on treatment outcome currently remains uncertain $[79,80]$. The gold standard in maxillofacial trauma imaging is multi-slice CT, but CBCT may be indicated if softtissue imaging is not needed $[40,81]$. CBCT can also be advantageous in evaluation of the radiological characteristics of bony pathoses of the mandible and maxilla before surgical intervention [40].

\subsection{Competency}

Since CBCT imaging has been in wide use for approximately 10-20 years, most working-age dentists are not familiar with СBCT equipment, image acquisition, or referral criteria. It is also noteworthy that there are significant differences between undergraduate curricula regarding radiation physics, radiation biology, safety and protection, and training in 3D-image interpretation. Continuing education is therefore essential and in many European countries, updating education in radiation protection is mandatory. As a DentoMaxilloFacial radiologist is a registered speciality only in a few countries in Europe, EADMFR has published a recommendation of core competencies and minimum training requirements for dentists involved with dental CBCT [82]. Two training levels are recommended; a basic level for dentists who prescribe CBCT scans, and an advanced level for the dentists who perform CBCT imaging and evaluate the $3 \mathrm{D}$ images. The aim is to ensure that dentists have adequate knowledge and skills in the justification and optimisation processes and CBCT image evaluation.

\section{Use of patient shields in dental CBCT}

Use of wearable radiation shields for patients during x-ray examinations is a tradition that dates back over 70 years [83]. The rationale for these protective measures is to reduce radiation exposure to the radiosensitive organs of patients and thereby improve radiological optimisation and radiation safety. This tradition has been embraced by both radiology professionals and patients and reinforced by regulators. However, significant development of medical imaging technology has brought new and more effective tools for optimisation, thereby providing enhanced radioprotection for patients in most of the x-ray imaging examinations and interventional procedures. This development has also been acknowledged in several scientific publications, policy statements, and guidelines over the past decade. Accordingly, the weight of evidence has gradually questioned the legacy use of personal patient shields [83-88]. The evidence brought by these studies suggest that contrary to the historical perspective that shielding is always beneficial to patients, shielding may have an adverse effect on imaging examination and may actually increase the radiation exposure to patients. In this review, we will not address this subject in depth. We will discuss what has been considered a consensus opinion to improve consistency in application of shielding in dental CBCT. We also discuss the use of thyroid shielding based on our Monte Carlo simulations that were reported in section 5.1. 

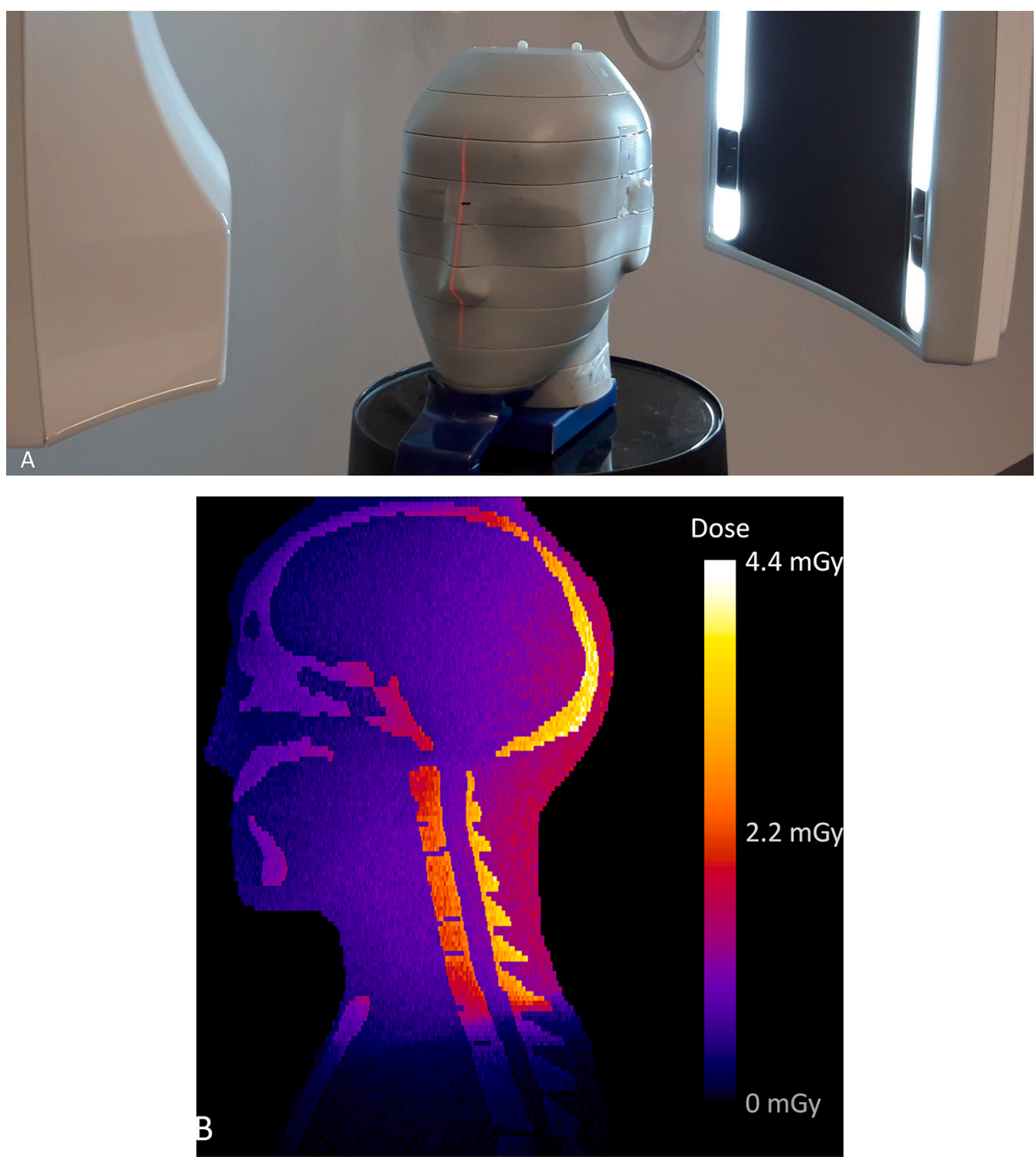

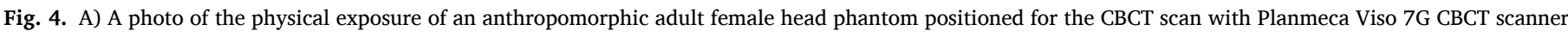

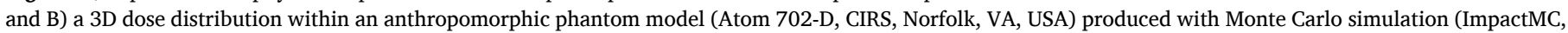

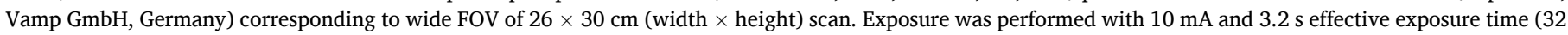

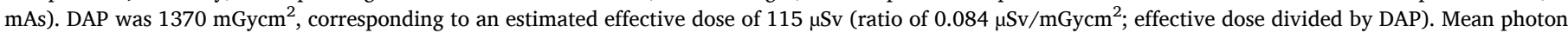

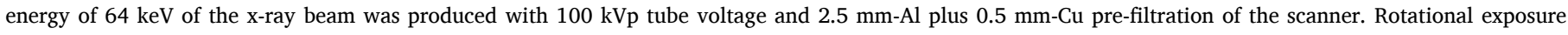
covered an angular range of $210^{\circ}$ in latero-posterior rotation (i.e. anterior part was not directly exposed).

In short, external radiation shields, mainly the thyroid collar shield, are not generally recommended in dental CBCT for radioprotection of patients, although different recommendations have also been presented $[83,89]$. The reasons for this alignment are as follows: 1) scan FOV should be optimised in all examinations, and thus the thyroid gland should not normally be in the primary x-ray beam, 2) use of a thyroid shield can easily interfere with the primary beam and cause significant artefacts, likely rendering diagnostically unacceptable images (as shown in Fig. 7), and 3) externally applied shielding is inefficient to protect radiosensitive organs from internal scatter. These reasons have also been discussed in a recent review by McGuigan et al. [90]. Radiation exposure from scatter to radiosensitive organs below the head and neck can always be assumed to be low in all dental $\mathrm{x}$-ray examinations regardless of specific modality and should not cause concern for e.g. pregnant patients [91]. As stated by the British Institute of Radiology in their recent and comprehensive patient shielding guidance, although there might be a slight benefit from thyroid shielding in dental CBCT studies with the largest FOVs, when consistent practices and risk of misplaced shields are considered, even this slight benefit can be seen as controversial [83].

To study the relative contribution of thyroid dose to effective dose, we performed Monte Carlo simulations as described in section 5.1. We found this contribution to be mainly below $10 \%$. The highest contributions (reaching $15 \%$ to $16 \%$ ) were related to scans of the mandible (lower jaw), where direct exposure is closest to the thyroid position. It is also such scans focused on the lower-jaw FOV where the risk of artefact from a thyroid shield is most pronounced. Higher contributions to effective dose from thyroid dose can be acquired with paediatric patients but also if the thyroid is partially or entirely in direct exposure (i.e. in the scan FOV). However, such FOV positioning should not occur with an appropriately performed CBCT scan. While considering the dental CBCT DRLs presented in Table 3 in combination with the conversion coefficients shown in Table 2, the typical dental CBCT indications imply an effective dose of approximately $50-100 \mu \mathrm{Sv}$. As such, the contribution of thyroid dose to effective dose can be assumed to be approximately 10 
Table 2

Conversion coefficients from DAP to effective dose based on Monte Carlo simulations. Conversion values cover three different FOV sizes (small, medium, large), three x-ray beam spectra ( $90 \mathrm{kV}$ with variable added filtrations: soft, medium, hard), different anatomical positions corresponding to main dental indications, and example of partial $200^{\circ}$ rotational exposure in third molar small FOV and whole skull large FOV scans in addition to default $360^{\circ}$ rotation. Mean photon energies in $\mathrm{keV}$ are marked in parentheses in three spectra used in simulations.

\begin{tabular}{|c|c|c|c|c|}
\hline & & $\begin{array}{l}\text { Spectra with } \\
90 \mathrm{kV}\end{array}$ & & \\
\hline \multirow[b]{2}{*}{ FOV } & \multirow[b]{2}{*}{ Location } & $\begin{array}{l}\text { Soft }(46.5 \\
\mathrm{keV})\end{array}$ & $\begin{array}{l}\text { Medium (54.9 } \\
\mathrm{keV} \text { ) }\end{array}$ & $\begin{array}{l}\text { Hard (65.3 } \\
\mathrm{keV})\end{array}$ \\
\hline & & $2.5 \mathrm{~mm}-\mathrm{Al}$ & $\begin{array}{l}2.5 \mathrm{~mm}-\mathrm{Al}+ \\
0.2 \mathrm{~mm}-\mathrm{Cu}\end{array}$ & $\begin{array}{l}2.5 \mathrm{~mm}-\mathrm{Al}+ \\
1.0 \mathrm{~mm}-\mathrm{Cu}\end{array}$ \\
\hline \multirow[t]{5}{*}{$\begin{array}{l}\text { Small }(3 \times 3 \\
\mathrm{cm})\end{array}$} & $\begin{array}{l}\text { Third Molar } \\
\left(360^{\circ}\right)\end{array}$ & 0.15176 & 0.22431 & 0.28592 \\
\hline & $\begin{array}{l}\text { Third Molar } \\
\left(200^{\circ}\right)\end{array}$ & 0.16687 & 0.24106 & 0.30310 \\
\hline & Canine (up) & 0.15354 & 0.23188 & 0.29903 \\
\hline & $\begin{array}{l}\text { Canine } \\
\text { (down) }\end{array}$ & 0.09780 & 0.16181 & 0.21921 \\
\hline & $\begin{array}{l}\text { Anterior } \\
\text { centre }\end{array}$ & 0.13703 & 0.20972 & 0.27303 \\
\hline \multirow{2}{*}{$\begin{array}{l}\text { Medium ( } 8 \\
\quad \times 8 \mathrm{~cm})\end{array}$} & Third Molar & 0.12726 & 0.19384 & 0.25099 \\
\hline & $\begin{array}{l}\text { Anterior } \\
\text { centre }\end{array}$ & 0.11438 & 0.17811 & 0.23340 \\
\hline \multirow{2}{*}{$\begin{array}{l}\text { Large }(20 \times \\
\quad 20 \mathrm{~cm})\end{array}$} & Skull $\left(360^{\circ}\right)$ & 0.06245 & 0.09721 & 0.12699 \\
\hline & Skull $\left(200^{\circ} \mathrm{s}\right)$ & 0.05798 & 0.09071 & 0.11917 \\
\hline
\end{tabular}

Table 3

Diagnostic reference levels of dentomaxillofacial CBCT examinations in various countries. Note that indications differ between the countries.

\begin{tabular}{|c|c|}
\hline Finland $[60]$ & $\begin{array}{l}\mathrm{KAP}, \mathrm{P}_{\mathrm{KA}} \\
\left.(\mathrm{mGycm})^{2}\right)\end{array}$ \\
\hline Presurgical imaging of implant treatments (single tooth) & 360 \\
\hline $\begin{array}{l}\text { Assessment of the relationship between a wisdom tooth and the } \\
\text { mandibular canal }\end{array}$ & 380 \\
\hline $\begin{array}{l}\text { Assessment of the periapical region and the root canal } \\
\text { morphology of the tooth }\end{array}$ & 550 \\
\hline Imaging of the paranasal sinuses (excluding traumas) & 1150 \\
\hline \multicolumn{2}{|l|}{ Sweden [61] } \\
\hline Part of jaw (1 quadrant) & $300 * / 150 * *$ \\
\hline Upper or lower jaw (2 quadrants) & $460 * / 180 * *$ \\
\hline Upper and lower jaw (4 quadrants) & $540 * / 260 * *$ \\
\hline \multicolumn{2}{|l|}{ Switzerland [59] } \\
\hline Wisdom tooth & 662 \\
\hline Single tooth implant & 683 \\
\hline Tooth position anomalies & 542 \\
\hline Pathological dentoalveolar modifications & 569 \\
\hline Endodontics & 639 \\
\hline \multicolumn{2}{|l|}{ UK $[62]$} \\
\hline $\begin{array}{l}\text { Adult (imaging prior to placement of a maxillary molar } \\
\text { implant) }\end{array}$ & 265 \\
\hline $\begin{array}{l}\text { Child (imaging of an impacted maxillary canine of a 12-year- } \\
\text { old child) }\end{array}$ & 170 \\
\hline $\mathrm{KAP}=\mathrm{P}_{\mathrm{KA}}=$ kerma-air product, *Upper level, **Lower level. & \\
\hline
\end{tabular}

$\mu \mathrm{Sv}$ or less in most CBCT examinations. Only a part of this thyroid dose contribution can be reduced with an externally placed thyroid shield and the remaining portion of thyroid dose is accumulated from internally scattered $\mathrm{x}$-rays without relevant means of avoidance. Therefore, although thyroid shielding has dose-reduction capability, the contribution of shielding in dental CBCT effective dose reduction can be regarded as fairly small and should be considered against the risk of artefacts caused by the shields. In summary, the use of external patient shields remains controversial [83,89], although modern technology with improving dose optimisation capabilities will gradually render traditional shielding obsolete [83].

\section{Future aspects}

\subsection{General development for optimisation}

In general, dental CBCT technology has shown incremental but steady development in many specific areas and applications during the past few years. New scanner models have already enabled a wider selection of different FOV sizes to optimise imaging for specific indications (minimising the volume of exposed tissue) and to accommodate a larger FOV to cover the whole cranial anatomy [14]. The adjustability of FOV size has also improved, with more seamless and flexible diameter and height value settings to accommodate any individual target size and patient-specific anatomy. This flexibility is important, not only for the indication and for patient-specific optimisation of radiation exposure, but also for an easier patient positioning and streamlined scan procedure.

\subsection{Reconstruction methods}

Reconstruction methods are steadily progressing further from the traditional 3D Feldkamp algorithm. Iterative reconstruction methods have complemented traditional algorithms and have potential for reduced image noise and artefacts in various $\mathrm{CT}$ and $\mathrm{CBCT}$ applications [18]. Iterative methods attempt to solve the main reconstruction inverse problem in various converging steps. Iterative algorithms include varying methods regarding the level of modelling of the actual scan from a technical and physical perspective. Iterative reconstruction can be done either in the image domain or at the raw data level. The basic principle of iterative reconstruction is to gradually (in iteration steps) update the image data such that the raw data, which is simulated (in forward-projection) from this image data, will approach the true (measured) raw-data projections [29]. Thus, when the difference between the simulated and the real raw data is sufficiently small (i.e. when the image data estimate will not change beyond the threshold value between the consecutive iteration steps), the iteration has reached the final solution. Iterative methods have shown a clear value in CT and CBCT imaging due to the flexibility of iterative methods to model various image acquisition geometries, and therefore provide more faithful assumptions of the actual image acquisition techniques [17]. The non-linearities built into iterative methods, mainly in the form of regularisation, have also revealed limitations of these methods as they may create images that deviate from traditional images on image texture, yielding even unnatural looking images [18]. This has raised concerns and has also highlighted the need for further development of reconstruction techniques [21].

The most recent advances in CT image reconstruction are focused in the realm of $\mathrm{AI}$, where the $\mathrm{DL}$ image reconstruction algorithms are showing promising results for image quality improvements [92]. Due to its nature, the DL iterative reconstruction algorithm is based on the learning data, which should represent a balanced distribution of clinically relevant sets of image data, including various indications. This means that such algorithms are much more detached from the original linear approximations of image reconstruction and as such require careful clinical validation to demonstrate reliability in specific clinical settings [21]. Due to its flexibility in various tasks also included in the radiological imaging process, the development and clinical implementation of DL algorithms in dental CBCT systems and clinical applications are likely to be significant factors in the upcoming years.

Regardless of the type of image reconstruction technique, the image reconstruction should also be able to perform corrections for metallic implants and involuntary patient movement during the scan. The challenge with patient involuntary movement during the scan is emphasised in dental CBCT, as the scan times are considerably (approximately an order of magnitude) longer than with multi-slice CT scanners. The challenge has already been addressed by existing motion artefact correction methods, which have been integrated into CBCT 

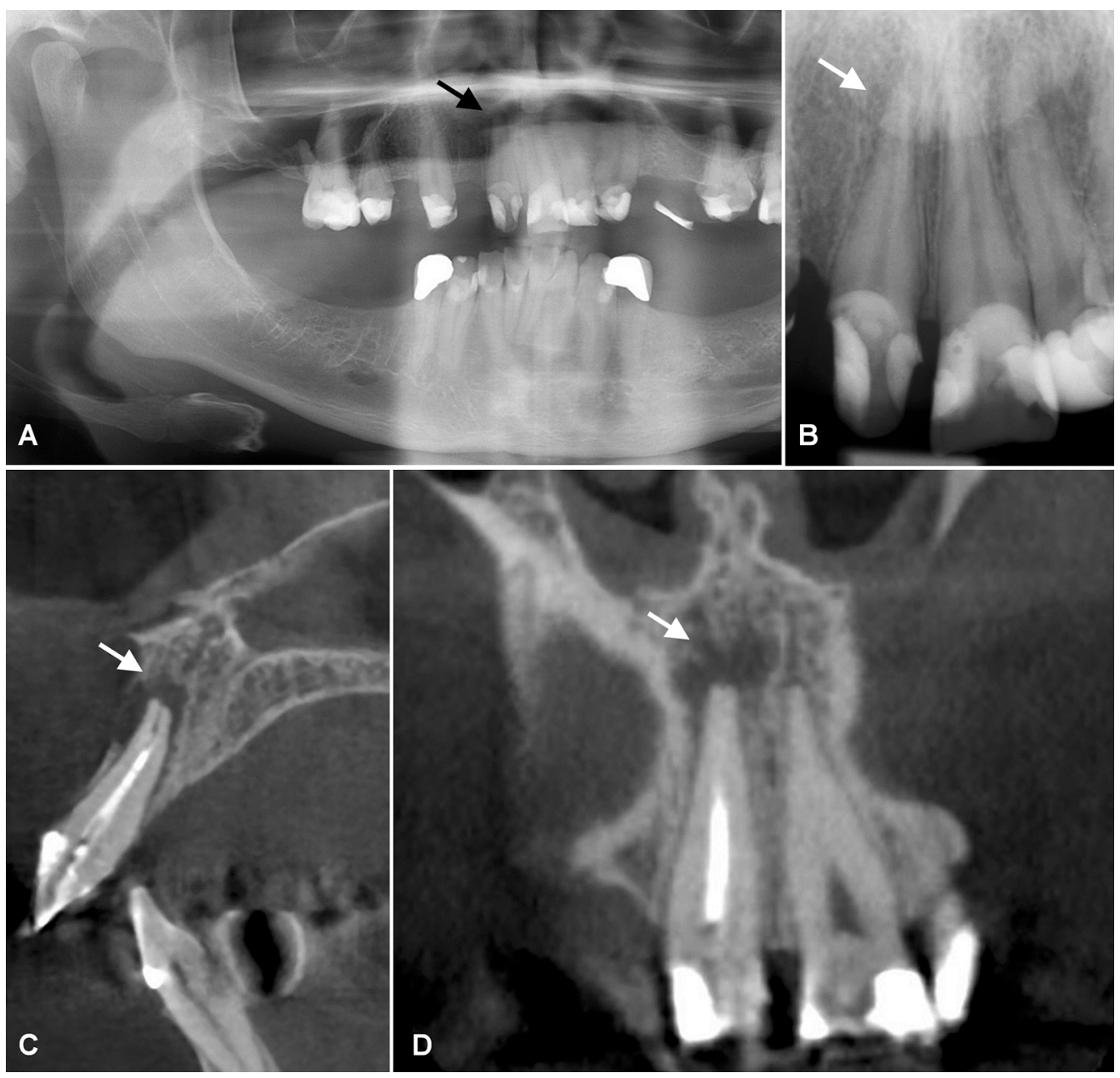

Fig. 5. Example clinical СВCT images in a case of apical periodontitis of the first maxillary incisor, presented in a) panoramic and b) intraoral images before rootcanal treatment and later c-d) in CBCT images during treatment.

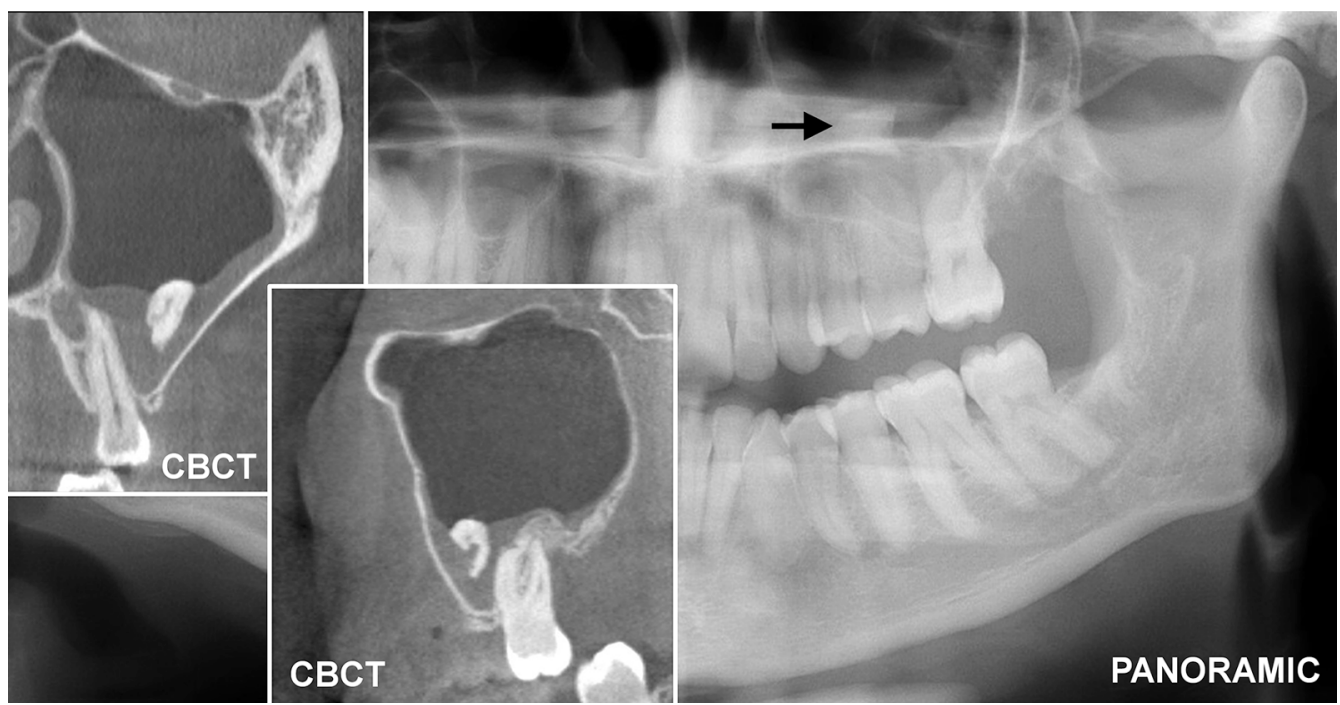

Fig. 6. Example clinical CBCT images from extraction of the maxillary wisdom tooth and the second molar, indicating postoperative complication. Root fragment in the left maxillary sinus is targeted in the panoramic image and CBCT images. 

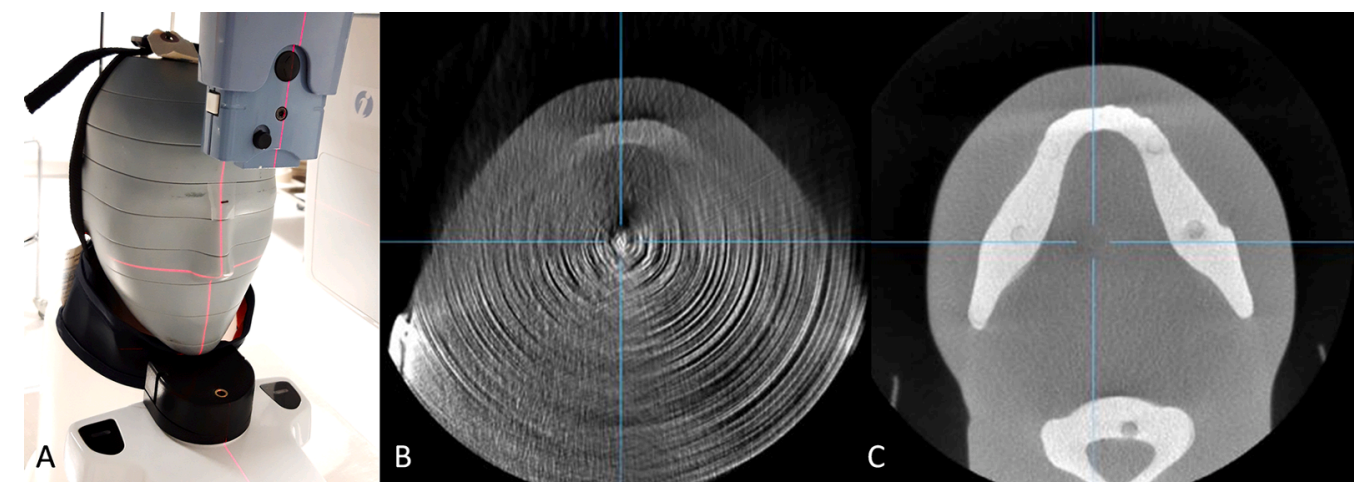

Fig. 7. Example of an artefact caused by an inappropriately positioned thyroid shield with respect to the $8 \times 15 \mathrm{~cm}$ FOV scanned with Instrumentarium OP300 CBCT scanner: a) a scan with an anthropomorphic head phantom with a thyroid shield positioned around the neck, b) an axial image with an artefact, c) an axial image without artefact when the scan was performed without a thyroid shield.

reconstruction for certain vendors [34]. An example of motion artefact correction in combination with noise reduction is presented in Fig. 8.

\subsection{Developing optimisation tools}

Radiation exposure in CT imaging has been recognised as an essential optimisation target for decades. Accordingly, there are many technical optimisation features available [93], also for dental CBCT scanners. Low-dose scan protocols may utilise increased beam filtration, enhanced reconstruction settings, larger voxel size, and minimised tube output, either with lower tube currents or with lower effective mAs as enabled with pulsed exposure. Possible further improvements may involve tube voltage reduction if allowed by increased $\mathrm{x}$-ray tube power, indication, and patient anatomy. This attempts to maximise the contrast in the image data by maximising the contribution of photoelectric effect at lower photon energies. Tube current modulation, which is routinely used in multi-slice CT scanners, may also be an efficient technique for optimisation in dental CBCT [6]. This technique aims to maintain a relatively constant net signal at the image detector for different raw-data projections of the image acquisition by producing tube current, which compensates for the changes in average projection-based attenuation of the patient while the $\mathrm{x}$-ray tube is rotating around the patient during the scan. Beam-shaping filters also attempt to achieve the same goal of equalising the detector signal by prefiltering the peripheral part of the $\mathrm{x}$ ray beam on every raw-data projection during the scan. This shaped prefilter has a shape of a bowtie and compensates the attenuation generated by the patient, assuming cylindrical patient geometry where the $\mathrm{x}$-ray projections passing through the central part of the patient attenuate more than the peripheral x-rays. In addition to detector signal equalisation, beam-shaping filters also help by reducing peripheral radiation exposure to the patient and improve image quality by reducing the amount of scatter from the peripheral anatomy. However, these potential optimisation methods, namely spectral optimisation, tube current modulation, and beam-shaping filters, require further development for dental CBCT hardware and x-ray production performance [5]. Finally, image detectors are becoming more sensitive in capturing the relevant image signal and reduce electronic noise by integrating the digital signal readout electronics more closely to the detector elements and via development of entirely new detector material and detector type developments, such as photon-counting detectors [93,94].

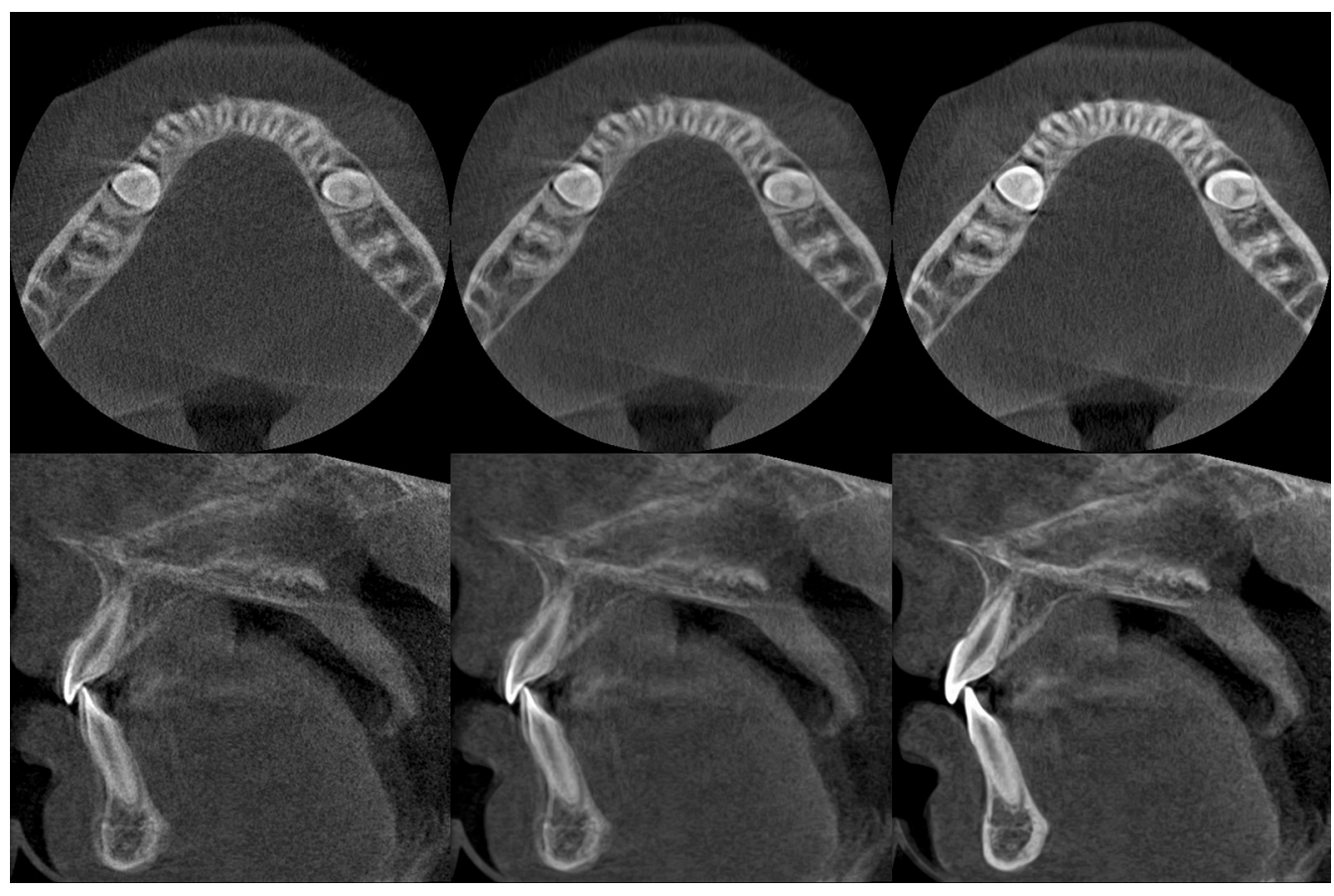

Fig. 8. Example of ultra-low dose patient images demonstrating the effect of CBCT noise reduction and motion artefact suppression algorithm in axial (upper row) and sagittal (lower row) reformats. Left: images with no corrections applied, Centre: images with noise reduction (AINO), Right: images with noise reduction and motion correction (CALM). Original images acquired with Planmeca ProMax Classic, $80 \times 80 \times 80$ $\mathrm{mm}$ FOV, $0.15-\mathrm{mm}$ voxel size, $90-\mathrm{kVp}$ tube voltage, and $18 \mathrm{mAs}$ exposure. Images courtesy of Planmeca Oy, Finland. 


\subsection{Photon-counting detectors}

Current CT and CBCT image detectors are energy-integrating detectors that accumulate the total energy delivered from absorbed $\mathrm{x}$-ray photons into measured signal intensity. Therefore, the detected signal does not reveal the characteristics of the applied photon spectrum. With photon-counting detectors, every absorbed photon releases electric charge carriers that are collected into measured charge signal as an individual detection event per each x-ray photon. This photon-energy specific signal amplitude can then be compared to pre-set threshold levels and are consequently counted into the corresponding photon energy bin. Depending on the number of threshold levels, which also reflect the complexity of the photon counting detector read-out electronics, multi-energy image data can be produced. Multi-energy image data may provide more advanced contrast representations (e.g. material-specific spectral imaging), yielding material density maps and virtual monochromatic images [94].

\subsection{General future aspects}

These new hardware- and software-based methods can be combined with significant potential to improve image quality and reduce radiation exposure to patients, as foreseen with future CBCT scanners. As these developments have already been implemented in conventional CT imaging and the speed of how these features are emerging in certain CT scanners (e.g. by AI algorithms and photon-counting detectors) also secure the future outlook for dental CBCT imaging. Despite these technological advancements, the value of new technology comes with welleducated and trained users who can take full benefit of the scanners and use them safely. This also involves multidisciplinary teams of physicists, radiologists, clinicians, and engineers, who should work together for more effective optimisation process in any medical imaging modality. Dental CBCT scanners have shown clear technical advancements in the past decade and scanner technology, models, and applications have diversified. This technical development is proceeding alongside new hardware and software methods, which complement each other. The main task for us is to understand this development and to know what is available in current CBCT scanners. Thus, we can make sound decisions what is needed for clinical practice and how to use our CBCT scanners in an optimal way for the benefit of the patient.

\section{Acknowledgements}

We acknowledge technologist Eliisa Leppänen from the HUS Medical Imaging Center for her collaboration in QA and anthropomorphic phantom CBCT scans with Instrumentarium OP300 scanner. We acknowledge Dr Mikko Lilja from Planmeca Oy for providing the motion correction clinical images. The authors declare no conflicts of interest. This research did not receive any grants from funding agencies in public, commercial, or governmental sector.

\section{References}

[1] Yoon DC, Mol A, Benn DK, Benavides E. Digital radiographic image processing and analysis. Dent Clin North Am 2018;62(3):341-59. https://doi.org/10.1016/j cden.2018.03.001.

[2] Boeddinghaus R, Whyte A. Current concepts in maxillofacial imaging. Eur J Radiol 2008;66(3):396-418. https://doi.org/10.1016/j.ejrad.2007.11.019.

[3] Nemtoi A, Czink C, Haba D, Gahleitner A. Cone beam CT: a current overview of devices. Dentomaxillofac Radiol 2013;42(8):20120443. https://doi.org/10.1259/ dmfr.20120443.

[4] Suomalainen A, Pakbaznejad Esmaeili E, Robinson S. Dentomaxillofacial imaging with panoramic views and cone beam CT. Insights Imaging 2015;6(1):1-16. https://doi.org/10.1007/s13244-014-0379-4.

[5] Kiljunen T, Kaasalainen T, Suomalainen A, Kortesniemi M. Dental cone beam CT: A review. Phys Med 2015;31(8):844-60. https://doi.org/10.1016/j. ejmp.2015.09.004.

[6] Jain S, Choudhary K, Nagi R, Shukla S, Kaur N, Grover D. New evolution of conebeam computed tomography in dentistry: Combining digital technologies. Imaging Sci Dent 2019;49:179-90. https://doi.org/10.5624/isd.2019.49.3.179.
[7] White SC, Pharoah MJ. The evolution and application of dental maxillofacial imaging modalities. Dent Clin North Am 2008;52:689-705. https://doi.org/ 10.1016/j.cden.2008.05.006. PMID: 18805224

[8] Hayashi T, Arai Y, Chikui T, Hayashi-Sakai S, Honda K, Indo H, et al. Clinical guidelines for dental cone-beam computed tomography. Oral Radiol 2018;34(2): 89-104. https://doi.org/10.1007/s11282-018-0314-3.

[9] Durack C, Patel S. Cone beam computed tomography in endodontics. Braz Dent J 2012;23(3):179-91. https://doi.org/10.1590/S0103-64402012000300001.

[10] Adibi S, Zhang W, Servos T, O'Neill PN. Cone beam computed tomography in dentistry: what dental educators and learners should know. J Dent Educ 2012;76 (11):1437-42. https://doi.org/10.1002/(ISSN)1930-783710.1002/jdd. 76.1110.1002/j.0022-0337.2012.76.11.tb05404.x.

[11] Mozzo P, Procacci C, Tacconi A, Tinazzi Martini P, Bergamo Andreis IA. A new volumetric CT machine for dental imaging based on the cone-beam technique: preliminary results. Eur Radiol 1998;8(9):1558-64. https://doi.org/10.1007/ s003300050586.

[12] Arai Y, Tammisalo E, Iwai K, Hashimoto K, Shinoda K. Development of a compact computed tomographic apparatus for dental use. Dentomaxillofac Radiol 1999;28: 245-8. https://doi.org/10.1038/sj/dmfr/4600448.

[13] Miracle AC, Mukherji SK. Conebeam CT of the head and neck, part 1: physical principles. AJNR Am J Neuroradiol 2009;30(6):1088-95. https://doi.org/ 10.3174/ajnr.A1653.

[14] Gaêta-Araujo H, Alzoubi T, Vasconcelos KdF, Orhan K, Pauwels R, Casselman JW, et al. Cone beam computed tomography in dentomaxillofacial radiology: a twodecade overview. Dentomaxillofac Radiol 2020;49(8):20200145. https://doi.org/ $10.1259 /$ dmfr. 20200145.

[15] Bushberg JT, Seibert JA, Leidholdt Jr E. The Essential Physics of Medical Imaging. 4th ed. Lippincott Williams \& Wilkins (LWW); 2020

[16] Feldkamp LA, Davis LC, Kress JW. Practical cone-beam algorithm. J Opt Soc Amer 1984;1:612-9. https://doi.org/10.1364/JOSAA.1.000612.

[17] Beister M, Kolditz D, Kalender WA. Iterative reconstruction methods in X-ray CT. Phys Med 2012;28(2):94-108. https://doi.org/10.1016/j.ejmp.2012.01.003.

[18] Stiller W. Basics of iterative reconstruction methods in computed tomography: a vendor-independent overview. Eur J Radiol 2018;109:147-54. https://doi.org/ 10.1016/j.ejrad.2018.10.025.

[19] Niebler S, Schömer E, Tjaden H, Schwanecke U, Schulze R. Projection-based improvement of 3D reconstructions from motion-impaired dental cone beam CT data. Med Phys 2019;46(10):4470-80. https://doi.org/10.1002/mp. v46.1010.1002/mp.13731.

[20] Wang G. A Perspective on Deep Imaging. IEEE Access 2016;4:8914-24. https://doi. org/10.1109/ACCESS.2016.2624938.

[21] Singh R, Wu W, Wang G, Kalra MK. Artificial intelligence in image reconstruction: The change is here. Phys Med 2020;79:113-25. https://doi.org/10.1016/j. ejmp.2020.11.012.

[22] Scarfe WC, Li Z, Aboelmaaty W, Scott SA, Farman AG. Maxillofacial cone beam computed tomography: essence, elements and steps to interpretation. Aust Dent J 2012;57(Suppl 1):46-60. https://doi.org/10.1111/j.1834-7819.2011.01657.x.

[23] Baba R, Ueda K, Okabe M. Using a flat-panel detector in high resolution cone beam CT for dental imaging. Dentomaxillofac Radiol 2004;33(5):285-90. https://doi. org $/ 10.1259 / \mathrm{dmfr} / 87440549$.

[24] Sheth NM, Zbijewski W, Jacobson MW, Abiola G, Kleinszig G, Vogt S, et al. Mobile C-Arm with a CMOS detector: Technical assessment of fluoroscopy and Cone-Beam CT imaging performance. Med Phys 2018;45:5420-36. https://doi.org/10.1002/ mp.13244.

[25] Pauwels R, Araki K, Siewerdsen JH, Thongvigitmanee SS. Technical aspects of dental CBCT: state of the art. Dentomaxillofac Radiol 2015;44(1):20140224. https://doi.org/10.1259/dmfr.20140224.

[26] Suomalainen A, Vehmas T, Kortesniemi M, Robinson S, Peltola J. Accuracy of linear measurements using dental cone beam and conventional multislice computed tomography. Dentomaxillofac Radiol 2008;37(1):10-7. https://doi.org/ $10.1259 / \mathrm{dmfr} / 14140281$

[27] Esmaeili F, Johari M, Haddadi P, Vatankhah M. Beam hardening artifacts: comparison between two cone beam computed tomography scanners. J Dent Res Dent Clin Dent Prospects 2012;6:49-53. https://doi.org/10.5681/joddd.2012.011.

[28] Barrett JF, Keat N. Artifacts in CT: recognition and avoidance. Radiographics 2004; 24(6):1679-91. https://doi.org/10.1148/rg.246045065.

[29] Hsieh J. Computed Tomography: Principles, Design, Artifacts, and Recent Advances. 2nd ed. Washington, USA: SPIE; 2009.

[30] Dang H, Stayman JW, Sisniega A, Zbijewski W, Xu J, Wang X, et al. Multiresolution statistical image reconstruction for mitigation of truncation effects: application to cone-beam CT of the head. Phys Med Biol 2017;62(2):539-59. https://doi.org/10.1088/1361-6560/aa52b8.

[31] Brüllmann D, Schulze RKW. Spatial resolution in CBCT machines for dental/ maxillofacial applications - what do we know today? Dentomaxillofac Radiol 2015;44(1):20140204. https://doi.org/10.1259/dmfr.20140204.

[32] RALPH WJ, JEFFERIES JR. The minimal width of the periodontal space. J Oral Rehabil 1984;11(5):415-8. https://doi.org/10.1111/jor.1984.11.issue-510.1111/ j.1365-2842.1984.tb00594.x.

[33] de Kinkelder R, Kalkman J, Faber DJ, Schraa O, Kok PHB, Verbraak FD, et al. Heartbeat-induced axial motion artifacts in optical coherence tomography measurements of the retina. Invest Ophthalmol Vis Sci 2011;52(6):3908. https:// doi.org/10.1167/iovs.10-6738.

[34] Santaella GM, Wenzel A, Haiter-Neto F, Rosalen PL, Spin-Neto R. Impact of movement and motion-artefact correction on image quality and interpretability in CBCT units with aligned and lateral-offset detectors. Dentomaxillofac Radiol 2020; 49(1):20190240. https://doi.org/10.1259/dmfr.20190240. 
[35] Ludlow JB, Timothy R, Walker C, Hunter R, Benavides E, Samuelson DB, et al. Effective dose of dental CBCT - a meta analysis of published data and additional data for nine CBCT units. Dentomaxillofac Radiol 2015;44(1):20140197. https:// doi.org/10.1259/dmfr.20140197.

[36] Mutalik S, Tadinada A, Molina MR, Sinisterra A, Lurie A. Effective doses of dental cone beam computed tomography: effect of 360-degree versus 180-degree rotation angles. Oral Surg Oral Med Oral Pathol Oral Radiol 2020;130(4):433-46. https:// doi.org/10.1016/j.0ooo.2020.04.008.

[37] Schulze R, Heil U, Gro $\beta$ D, Bruellmann DD, Dranischnikow E, Schwanecke U, et al. Artefacts in CBCT: a review. Dentomaxillofac Radiol 2011;40(5):265-73. https:// doi.org/10.1259/dmfr/30642039.

[38] Deutsches Institut für Normung. DIN 6868-15: Image quality assurance in X-ray departments - Part 15: RöV constancy testing of X-ray installations for dental radiographic equipment for digital cone-beam computed tomography. Berlin: Deutsches Institut für Normung; 2015 [in German].

[39] Deutsches Institut für Normung. DIN 6868-161: Image quality assurance in X-ray departments - Part 161: RöV acceptance testing of dental radiographic equipmen for digital cone beam computed tomography. English translation of DIN 6868-161: 2013-01. Berlin: Deutsches Institut für Normung; 2016. Original and English translation available at https://www.beuth.de/en/standard/din-6868-161/ 164214522.

[40] European Commission. Radiation protection 172. Cone beam CT for dental and maxillofacial radiology (Evidence-based guidelines). Luxembourg: Office for Official Publications of the European Communities; 2012. https://ec.europa.eu/ energy/sites/ener/files/documents/172.pdf [accessed 2 Feb 2021].

[41] de Las Heras Gala H, Torresin A, Dasu A, Rampado O, Delis H, Hernández Girón I, et al. Quality control in cone-beam computed tomography (CBCT) EFOMP-ESTROIAEA protocol. 2nd Edition, May 2019. https://www.efomp.org/uploads/ 2d23d153-b77c-4161-802c-5b8422d15e29/EFOMP_IAEA_ESTRO_\%20CBCT 2019_05_27.pdf [accessed 2 Feb 2021].

[42] International Commission on Radiation Protection (ICRP). Recommendations of the International Commission on Radiological Protection. ICRP Publication 26. Ann ICRP 1977;1.

[43] European Commission, Food and Agriculture Organizations of the United Nations, International Atomic Energy Agency, International Labour Organization, OECD Nuclear Energy Agency, PAN American Health Organization, United Nations Environment programme, World Health Organization. Radiation Protection and Safety of Radiation Sources: International Basic Safety Standards. IAEA Safety Standards Series No. GSR Part 3, IAEA, Vienna (2014). https://www-pub.iaea.org/ MTCD/publications/PDF/Pub1578_web-57265295.pdf. [accessed 2 Feb 2021].

[44] Council Directive 2013/59/Euratom of 5 December 2013 laying down basic safety standards for protection against the dangers arising from exposure to ionising radiation, and repealing Directives 89/618/Euratom, 90/641/Euratom, 96/29/ Euratom, 97/43/. Official Journal of the European Union. L 2014 https://eur-lex europa.eu/LexUriServ/LexUriServ.do?uri=OJ:L:2014:013:0001:0073:EN:PDF [accessed 2 Feb 2021]

[45] Yeung AWK, Jacobs R, Bornstein MM. Novel low-dose protocols using cone beam computed tomography in dental medicine: a review focusing on indications, limitations, and future possibilities. Clin Oral Investig 2019;23(6):2573-81. https://doi.org/10.1007/s00784-019-02907-y.

[46] International Commission on Radiation Protection (ICRP). Radiological Protection in Cone Beam Computed Tomography (CBCT). ICRP Publication 129. Ann ICRP 2015;44(1).

[47] Siiskonen T, Gallagher A, Ciraj Bjelac O, Novak L, Sans Merce M, Farah J, et al. A European perspective on Dental Cone Beam Computed Tomography (CBCT) systems with a focus on optimisation utilising DRLs (Diagnostic Reference Levels). J Radiol Prot 2021 doi: 10.1088/1361-6498/abdd05. Epub ahead of print.

[48] Bornstein MM, Scarfe WC, Vaughn VM, Jacobs R. Cone beam computed tomography in implant dentistry: a systematic review focusing on guidelines, indications, and radiation dose risks. Int J Oral Maxillofac Implants 2014;29 (Suppl):55-77. https://doi.org/10.11607/jomi.2014suppl.g1.4.

[49] Mah E, Ritenour ER, Yao H. A review of dental cone-beam CT dose conversion coefficients. Dentomaxillofacial Radiol 2021;50(3):20200225. https://doi.org/ 10.1259/dmfr.20200225.

[50] Oenning AC, Jacobs R, Pauwels R, Stratis A, Hedesiu M, Salmon B. Cone-beam CT in paediatric dentistry: DIMITRA project position statement. Pediatr Radiol 2018; 48(3):308-16. https://doi.org/10.1007/s00247-017-4012-9.

[51] Beganović A, Ciraj-Bjelac O, Dyakov I, Gershan V, Kralik I, Milatović A, et al. IAEA survey of dental cone beam computed tomography practice and related patient exposure in nine Central and Eastern European countries. Dentomaxillofac Radiol 2020;49(1):20190157. https://doi.org/10.1259/dmfr.20190157.

[52] (EFOMP 2017) de Las Heras Gala H, Torresin A, Dasu A, Rampado O, Delis H, Hernández Girón I, et al. Quality control in cone-beam computed tomography (CBCT) EFOMP-ESTRO-IAEA protocol (summary report). Phys Med 2017;39:67-72. doi: 10.1016/j.ejmp.2017.05.069.

[53] Koivisto J, Kiljunen T, Tapiovaara M, Wolff J, Kortesniemi M. Assessment of radiation exposure in dental cone-beam computerized tomography with the use of metal-oxide semiconductor field-effect transistor (MOSFET) dosimeters and Monte Carlo simulations. Oral Surg Oral Med Oral Pathol Oral Radiol 2012;114(3): 393-400. https://doi.org/10.1016/j.00oo.2012.06.003.

[54] Li Y, Huang B, Cao J, Fang T, Liu G, Li X, et al. Estimating radiation dose to major organs in dental X-ray examinations: a phantom study. Radiat Prot Dosim 2020; 192:328-34. doi: 10.1093/rpd/ncaa196.

[55] Lee C, Yoon J, Han S-S, Na JY, Lee J-H, Kim YH, et al. Dose assessment in dental cone-beam computed tomography: comparison of optically stimulated luminescence dosimetry with Monte Carlo method. PLoS ONE 2020;15(3): e0219103. https://doi.org/10.1371/journal.pone.021910310.1371/journal. pone.0219103.g00110.1371/journal.pone.0219103.g00210.1371/journal. pone.0219103.g00310.1371/journal.pone.0219103.g00410.1371/journal. pone.0219103.g00510.1371/journal.pone.0219103.t00110.1371/journal. pone.0219103.t00210.1371/journal.pone.0219103.t00310.1371/journal. pone.0219103.t00410.1371/journal.pone.0219103.s001.

[56] Morant JJ, Salvadó M, Casanovas R, Hernández-Girón I, Velasco E, Calzado A. Validation of a Monte Carlo simulation for dose assessment in dental cone beam CT examinations. Phys Med 2012;28(3):200-9. https://doi.org/10.1016/j. ejmp.2011.06.047.

[57] Oenning AC, Pauwels R, Stratis A, De Faria Vasconcelos K, Tijskens E, De Grauwe A, et al. Halve the dose while maintaining image quality in paediatric Cone Beam CT. Sci Rep 2019;9(1). https://doi.org/10.1038/s41598-019-41949-w.

[58] Tsapaki V. Radiation protection in dental radiology - Recent advances and future directions. Phys Med 2017;44:222-6. https://doi.org/10.1016/j. ejmp.2017.07.018.

[59] Deleu M, Dagassan D, Berg I, Bize J, Dula K, Lenoir V, et al. Establishment of national diagnostic reference levels in dental cone beam computed tomography in Switzerland. Dentomaxillofac Radiol 2020;49(6):20190468. https://doi.org/ $10.1259 / \mathrm{dmfr} .20190468$.

[60] STUK's decision STUK S/4/2019, Säteilyturvakeskuksen määräys oikeutusarvioinnista ja säteilysuojelun optimoinnista lääketieteellisessä altistuksessa. (In Finnish) www.stuklex.fi/fi/maarays/stuk-s-4-2019. [accessed 2 Feb 2021].

[61] Strålsäkerhetsmyndighetens föreskrifter och allmänna råd om medicinska exponeringar. Strålsäkerhetsmyndigheten (SSM). SSMFS 2018:5. (In Swedish) https://www.stralsakerhetsmyndigheten.se/publikationer/foreskrifter/ssmfs2018/ssmfs-20185/ [accessed 2 Feb 2021].

[62] Holroyd JR, Smith JRH, Edyvean S. PHE-CRCE-59: Dose to patients from dental radiographic X-ray imaging procedures in the UK - 2017 Review. Public Health England (PHE) 2020. Gateway Number: GW-1261. https://assets.publishing. service.gov.uk/government/uploads/system/uploads/attachment_data/file/ 918078/2019 dental_NDRL_report.pdf. [accessed 2 Feb 2021].

[63] American Dental Association Council on Scientific Affairs. The use of cone-beam computed tomography in dentistry: an advisory statement from the American Dental Association Council on Scientific Affairs. J Am Dent Assoc 2012;143:899092. doi: 10.14219/jada.archive.2012.0295.

[64] Pauwels R. Cone beam CT for dental and maxillofacial imaging: dose matters. Radiat Prot Dosimetry 2015;165:156-61. https://doi.org/10.1093/rpd/ncv057.

[65] Carter L, Farman AG, Geist J, Scarfe WC, Angelopoulos C, Nair MK, et al. American Academy of Oral and Maxillofacial Radiology executive opinion statement on performing and interpreting diagnostic cone beam computed tomography. Oral Surg Oral Med Oral Pathol Oral Radiol Endod 2008;106(4):561-2. https://doi.org/ 10.1016/j.tripleo.2008.07.007.

[66] Horner K, Islam M, Flygare L, Tsiklakis K, Whaites E. Basic principles for use of dental cone beam computed tomography: consensus guidelines of the European Academy of Dental and Maxillofacial Radiology. Dentomaxillofac Radiol 2009;38 (4):187-95. https://doi.org/10.1259/dmfr/74941012.

[67] Kim IH, Singer SR, Mupparapu M. Review of cone beam computed tomography guidelines in North America. Quintessence Int 2019;50:136-45. https://doi.org/ 10.3290/j.qi.a41332.

[68] Matzen LH, Berkhout E. Cone beam CT imaging of the mandibular third molar: a position paper prepared by the European Academy of DentoMaxilloFacial Radiology (EADMFR). Dentomaxillofac Radiol 2019;48(5):20190039. https://doi. org/10.1259/dmfr.20190039.

[69] Horner K, Barry S, Dave M, Dixon C, Littlewood A, Pang CL, et al. Diagnostic efficacy of cone beam computed tomography in paediatric dentistry: a systematic review. Eur Arch Paediatr Dent 2020;21(4):407-26. https://doi.org/10.1007/ s40368-019-00504-X.

[70] De Grauwe A, Ayaz I, Shujaat S, Dimitrov S, Gbadegbegnon L, Vande Vannet B, et al. CBCT in orthodontics: a systematic review on justification of CBCT in a paediatric population prior to orthodontic treatment. Eur J Orthod 2019;41:381-9. doi: 10.1093/ejo/cjy066.

[71] American Academy of Oral and Maxillofacial Radiology. Clinical recommendations regarding use of cone beam computed tomography in orthodontics. [corrected]. Position statement by the American Academy of Oral and Maxillofacial Radiology. Oral Surg Oral Med Oral Pathol Oral Radiol 2013;116:238-57. doi: 10.1016/j. oooo.2013.06.002.

[72] Kapila SD, Nervina JM. CBCT in orthodontics: assessment of treatment outcomes and indications for its use. Dentomaxillofac Radiol 2015;44(1):20140282. https:// doi.org/10.1259/dmfr.20140282.

[73] Patel S, Durack C, Abella F, Roig M, Shemesh H, Lambrechts P, et al. European Society of Endodontology position statement: the use of CBCT in endodontics. Int Endod J 2014;47(6):502-4. https://doi.org/10.1111/iej.2014.47.issue-610.1111/ iej.12267.

[74] Patel S, Brown J, Semper M, Abella F, Mannocci F. European Society of Endodontology position statement: Use of cone beam computed tomography in Endodontics: European Society of Endodontology (ESE) developed by Int Endod J 2019;52(12):1675-8. https://doi.org/10.1111/iej.v52.1210.1111/iej.13187.

[75] Turnbull-Smith SB. Cone-Beam Computed Tomography Examinations of the Head and Neck Region in Finland: Indications and Patient Radiation Dose. Tampere University of Technology 2016 http://urn.fi/URN:NBN:fi:tty-201608264465. [accessed 28.12.2020].

[76] Tyndall DA, Price JB, Tetradis S, Ganz SD, Hildebolt C, Scarfe WC, et al. Position statement of the American Academy of Oral and Maxillofacial Radiology on selection criteria for the use of radiology in dental implantology with emphasis on 
cone beam computed tomography. Oral Surg Oral Med Oral Pathol Oral Radiol 2012;113:817-26. https://doi.org/10.1016/j.oooo.2012.03.005.

[77] Bornstein MM, Horner K, Jacobs R. Use of cone beam computed tomography in implant dentistry: current concepts, indications and limitations for clinical practice and research. Periodontol 2000;2017(73):51-72. https://doi.org/10.1111/ prd.12161.

[78] Jacobs R, Salmon B, Codari M, Hassan B, Bornstein MM. Cone beam computed tomography in implant dentistry: recommendations for clinical use. BMC Oral Health 2018;18:88. https://doi.org/10.1186/s12903-018-0523-5.

[79] Larheim TA, Abrahamsson A-K, Kristensen M, Arvidsson LZ. Temporomandibular joint diagnostics using CBCT. Dentomaxillofac Radiol 2015;44(1):20140235. https://doi.org/10.1259/dmfr.20140235.

[80] Weiss 2nd R, Read-Fuller A. Cone Beam Computed Tomography in Oral and Maxillofacial Surgery: An Evidence-Based Review. Dent J (Basel) 2019;7:52. https://doi.org/10.3390/dj7020052.

[81] Carter JB, Stone JD, Clark RS, Mercer JE. Applications of Cone-Beam Computed Tomography in Oral and Maxillofacial Surgery: An Overview of Published Indications and Clinical Usage in United States Academic Centers and Oral and Maxillofacial Surgery Practices. J Oral Maxillofac Surg 2016;74(4):668-79. https://doi.org/10.1016/j.joms.2015.10.018.

[82] Brown J, Jacobs R, Levring Jäghagen E, Lindh C, Baksi G, Schulze D, et al. Basic training requirements for the use of dental CBCT by dentists: a position paper prepared by the European Academy of DentoMaxilloFacial Radiology. Dentomaxillofac Radiol 2014;43(1):20130291. https://doi.org/10.1259/ dmfr.20130291.

[83] British Institute of Radiology. Guidance on using shielding on patients for diagnostic radiology applications. BIR, London, UK; 2020. https://www.bir.org. uk/media/416143/final_patient_shielding_guidance.r1.pdf (accessed 2 Feb 2021).

[84] Fawcett SL, Gomez AC, Barter SJ, Ditchfield M, Set P. More harm than good? The anatomy of misguided shielding of the ovaries. Br J Radiol 2012;85(1016):e442-7. https://doi.org/10.1259/bjr/25742247.

[85] American Association of Physicists in Medicine (AAPM). AAPM Position Statement on the use of Bismuth shielding for the purpose of dose reduction in CT scanning.
Policy Number PP 26-B;2017. https://www.aapm.org/publicgeneral/ BismuthShielding.pdf. (accessed 16 Jan 2021).

[86] American Association of Physicists in Medicine (AAPM). AAPM Position Statement on the Use of Patient Gonadal and Fetal Shielding. Policy Number PP 32-A; 2019.

[87] Marsh RM, Silosky M. Patient shielding in diagnostic imaging: discontinuing a legacy practice. AJR Am J Roentgenol 2019;212(4):755-7. https://doi.org/ 10.2214/AJR.18.20508.

[88] McKenney S, Gingold E, Zaidi H. Gonad shielding should be discontinued for most diagnostic imaging exams. Med Phys 2019;46:1111-4. doi: 10.1002/mp.13409. https://www.aapm.org/org/policies/details.asp? id=468\&type=PP\&current=true. [accessed 2 Feb 2021].

[89] Pauwels R, Horner K, Vassileva J, Rehani MM. Thyroid shielding in cone beam computed tomography: recommendations towards appropriate use. Dentomaxillofac Radiol 2019;48(7):20190014. https://doi.org/10.1259/ dmfr.20190014.

[90] McGuigan MB, Duncan HF, Horner K. An analysis of effective dose optimization and its impact on image quality and diagnostic efficacy relating to dental cone beam computed tomography (CBCT). Swiss Dent J 2018;128:297-316.

[91] Kelaranta A, Ekholm M, Toroi P, Kortesniemi M. Radiation exposure to foetus and breasts from dental X-ray examinations: effect of lead shields. Dentomaxillofac Radiol 2016;45(1):20150095. https://doi.org/10.1259/dmfr.20150095.

[92] McCollough CH, Leng S. Use of artificial intelligence in computed tomography dose optimisation. Ann ICRP 2020;49(1_suppl):113-25. https://doi.org/10.1177/ 0146645320940827.

[93] McCollough CH, Chen GH, Kalender W, Leng S, Samei E, Taguchi K, et al. Achieving routine submillisievert CT scanning: report from the summit on management of radiation dose in CT. Radiology 2012;264(2):567-80. https://doi. org/10.1148/radiol.12112265.

[94] Flohr T, Petersilka M, Henning A, Ulzheimer S, Ferda J, Schmidt B. Photoncounting CT review. Phys Med 2020;79:126-36. https://doi.org/10.1016/j. ejmp.2020.10.030. 